\title{
Physiological and Biochemical Responses of Ageratum conyzoides, Oryza sativa f. spontanea (Weedy Rice) and Cyperus iria to Parthenium hysterophorus Methanol Extract
}

\author{
Mst. Motmainna ${ }^{1}$, Abdul Shukor Juraimi ${ }^{1, *}$, Md. Kamal Uddin ${ }^{2}$, Norhayu Binti Asib ${ }^{3}$, \\ A. K. M. Mominul Islam ${ }^{4}\left(\mathbb{D}\right.$, Muhammad Saiful Ahmad-Hamdani ${ }^{1}{ }^{1}$, Zulkarami Berahim $^{5}{ }^{(\mathbb{C}}$ \\ and Mahmudul Hasan ${ }^{1}$ \\ check for \\ updates \\ 1 Department of Crop Science, Faculty of Agriculture, Universiti Putra Malaysia, \\ Serdang 43400, Selangor, Malaysia; gs51794@student.upm.edu.my (M.M.); \\ s_ahmad@upm.edu.my (M.S.A.-H.); gs53801@student.upm.edu.my (M.H.) \\ 2 Department of Land Management, Faculty of Agriculture, Universiti Putra Malaysia, \\ Serdang 43400, Selangor, Malaysia; mkuddin@upm.edu.my \\ 3 Department of Plant Protection, Faculty of Agriculture, Universiti Putra Malaysia, \\ Serdang 43400, Selangor, Malaysia; norhayuasib@upm.edu.my \\ 4 Department of Agronomy, Faculty of Agriculture, Bangladesh Agricultural University, \\ Mymensingh 2202, Bangladesh; akmmominulislam@bau.edu.bd \\ 5 Laboratory of Climate-Smart Food Crop Production, Institute of Tropical Agriculture and Food Security, \\ Universiti Putra Malaysia, Serdang 43400, Selangor, Malaysia; zulkerami@upm.edu.my \\ * Correspondence: ashukur@upm.edu.my
}

Citation: Motmainna, M..; Juraimi, A.S.; Uddin, M..K.; Asib, N.B.; Islam, A.K.M.M.; Ahmad-Hamdani, M.S.; Berahim, Z.; Hasan, M. Physiological and Biochemical Responses of Ageratum conyzoides, Oryza sativa f. spontanea (Weedy Rice) and Cyperus iria to Parthenium hysterophorus Methanol Extract. Plants 2021, 10, 1205. https://doi.org/10.3390/ plants10061205

Academic Editor: Adela M. Sánchez Moreiras

Received: 1 March 2021

Accepted: 13 April 2021

Published: 14 June 2021

Publisher's Note: MDPI stays neutral with regard to jurisdictional claims in published maps and institutional affiliations.

Copyright: (c) 2021 by the authors. Licensee MDPI, Basel, Switzerland. This article is an open access article distributed under the terms and conditions of the Creative Commons Attribution (CC BY) license (https:// creativecommons.org/licenses/by/ $4.0 /)$.
Abstract: The current study was designed to investigate the effect of Parthenium hysterophorus L. methanol extract on Ageratum conyzoides L., Oryza sativa f. spontanea (weedy rice) and Cyperus iria L. in glasshouse condition. Here, Parthenium hysterophorus methanol extract at 20, 40, and $60 \mathrm{~g} \mathrm{~L}^{-1}$ concentrations was applied on the test species to examine their physiological and biochemical responses at 6, 24, 48 and $72 \mathrm{~h}$ after spraying (HAS). The phytotoxicity of $P$. hysterophorus was strong on A. conyzoides compared to weedy rice and Cyperus iria at different concentrations and exposure times. There was a reduction in photosynthesis rate, stomatal conductance, transpiration, chlorophyll content and carotenoid content when plants were treated with P. hysterophorus extract concentrations. Exposure to P. hysterophorus $\left(60 \mathrm{~g} \mathrm{~L}^{-1}\right)$ at 24 HAS increased malondialdehyde (MDA) and proline content by $152 \%$ and $130 \%$, respectively, in A. conyzoides compared with control. The activities of antioxidant enzymes (superoxide dismutase (SOD), catalase (CAT) and peroxidase (POD)) were also increased in the presence of $P$. hysterophorus extract. Present findings confirm that the methanol extract of $P$. hysterophorus can disrupt the physiological and biochemical mechanism of target weeds and could be used as an alternative to chemical herbicides.

Keywords: P. hysterophorus; photosynthesis; chlorophyll; antioxidant enzymes; malondialdehyde; proline; A. conyzoides

\section{Introduction}

Weeds sometimes compete with crops for space, nutrients, water, and light, resulting in growth reduction and yield loss of cultivated crops. Ageratum conyzoides (L.) L., Oryza sativa f. spontane Roshev. (weedy rice) and Cyperus iria L. are common weeds in the cultivated lands. Ageratum conyzoides could be considered an aggressive weed and can infest cultivated agricultural land and cause a severe yield loss by interfering growth and development of different crops [1]. Weedy rice possesses similar characteristics to cultivated rice and has a long unsettled problem in rice granaries [2]. It has been reported that weedy rice caused yield loss in rice fields from $16 \%$ to $74 \%$ in Asia [3-5]. Cyperus iria is an annual herbaceous sedge and responsible for reducing crop yield, particularly in rice and caused a $64 \%$ reduction in rice yield [6]. 
Parthenium hysterophorus is considered an invasive weed, which belongs to the Asteraceae family. It creates severe problems in human health, crop production and animal husbandry [7]. High germination rate, prolific nature and resistance to chemical herbicide are the major challenges to control this noxious weed. The allelopathic potential of certain weed and crop species can influence the growth and distribution of associated weed species and the yield of desired plants. Moreover, allelopathy has been employed successfully in biocontrol programs focusing on the control of problematic weeds and plant diseases [8]. Previous reports have shown that $P$. hysterophorus has the allelopathic potential [8-10], which could be introduced in the weed management program.

Various physiological and biochemical responses, including chlorosis, lipid peroxidation and antioxidant responses, can occur in plants as a consequence of herbicide exposure [11]. Chemical stress is abiotic stress that obstructs a plant's growth and, when applied in high concentrations, could be lethal. Abiotic and biotic conditions dictate the physiological functions and stability of the plant. However, various abiotic stresses lead to the overproduction of reactive oxygen species (ROS) in plants, which are highly reactive and toxic, causing damage to proteins, lipids, carbohydrates and DNA, which ultimately results in oxidative stress [12]. Chlorophylls are the fundamental pigments that play a key role in photosynthesis. Carotenoids play an important photo-protective role by scavenging ROS and suppressing lipid peroxidation [13]. Differences in plant leaf chlorophyll and carotenoid content reflect their resistance levels and photosynthetic capacities [14]. Under oxidative stress, plants respond by increasing antioxidant defenses, particularly enzymes such as superoxide dismutase (SOD), peroxidase (POD) and catalase (CAT) [15]. SOD happens in several cell compartments and catalyzes the dismutation of the superoxide anion into hydrogen peroxide $\left(\mathrm{H}_{2} \mathrm{O}_{2}\right)$ and molecular oxygen $\left(\mathrm{O}_{2}\right)$ [16]. Hydrogen peroxide, in turn, is removed by several other antioxidant enzymes, such as CAT and POD [17]. Malondialdehyde (MDA) is a metabolic product of lipid peroxidation and a widely accepted indicator of oxidative damage in plants [18]. Lipid peroxidation affects the physiological process of the cell [19].

Many studies are available on allelopathy, but not much is known about its mechanism of activity. This study aims to determine physiological and biochemical changes resulting from the implementation of $P$. hysterophorus extract concentration on $A$. conyzoides, weedy rice and C. iria. An understanding of the physiological and biochemical phenomenon of allelopathy will no doubt explicate the mechanism of action and the possible target site. Thus an investigation into the allelopathic potential of $P$. hysterophorus was conducted.

\section{Results}

\subsection{Effect of P. hysterophorus Extract on Chlorophyll-a Content of the Test Plant Species}

Foliar spray of $P$. hysterophorus extract had a significant effect on chlorophyll- $a$ content of A. conyzoides, weedy rice and C. iria at 6, 24, 48 and $72 \mathrm{~h}$ after spray (HAS) (Table 1). Chlorophyll-a content showed a decline from 12.50 to $31.98 \%, 30.01$ to $74.32 \%, 29.78$ to $73.53 \%$ and 31.10 to $74.09 \%$ in $A$. conyzoides compared to control $\left(0 \mathrm{~g} \mathrm{~L}^{-1}\right)$ at $6,24,48$, and 72 HAS, respectively by foliar application of P. hysterophorus at different concentrations. Simultaneously, only a $36 \%$ decline was observed in weedy rice and C. iria after $24 \mathrm{~h}$ at the highest concentration $\left(60 \mathrm{~g} \mathrm{~L}^{-1}\right)$ of P. hysterophorus extract. 
Table 1. Response of chlorophyll- $a$ content and chlorophyll- $b$ of $A$. conyzoides, weedy rice and C. iria to a foliar spray of P. hysterophorus extract.

\begin{tabular}{|c|c|c|c|c|c|c|c|c|c|}
\hline \multirow{3}{*}{ Test Plants } & \multirow{3}{*}{$\begin{array}{l}\text { Dose } \\
\left(\mathrm{g} \mathrm{L}^{-1}\right)\end{array}$} & \multicolumn{4}{|c|}{ Chlorophyll- $a\left(\mathrm{mg} \mathrm{g}^{-1} \mathrm{FW}\right)$} & \multicolumn{4}{|c|}{ Chlorophyll-b (mg g $\left.{ }^{-1} \mathrm{FW}\right)$} \\
\hline & & \multicolumn{4}{|c|}{ Hours after Spray } & \multicolumn{4}{|c|}{ Hours after Spray } \\
\hline & & 6 & 24 & 48 & 72 & 6 & 24 & 48 & 72 \\
\hline \multirow{4}{*}{$\begin{array}{c}\text { A. } \\
\text { conyzoides }\end{array}$} & 0 & $\begin{array}{c}3.03 a \pm 0.04 \\
(0)\end{array}$ & $\begin{array}{c}3.05 \mathrm{a} \pm 0.06 \\
(0)\end{array}$ & $\begin{array}{c}2.91 \mathrm{a} \pm 0.11 \\
(0)\end{array}$ & $\begin{array}{c}3.06 \mathrm{a} \pm 0.07 \\
(0)\end{array}$ & $\begin{array}{c}1.14 \mathrm{a} \pm 0.08 \\
(0)\end{array}$ & $\begin{array}{c}1.16 \mathrm{a} \pm 0.09 \\
(0)\end{array}$ & $\begin{array}{c}1.11 \mathrm{a} \pm 0.12 \\
(0)\end{array}$ & $\begin{array}{c}1.31 \mathrm{a} \pm 0.13 \\
(0)\end{array}$ \\
\hline & 20 & $\begin{array}{c}2.65 a \pm 0.11 \\
(12.50)\end{array}$ & $\begin{array}{c}2.13 b \pm 0.07 \\
(30.01)\end{array}$ & $\begin{array}{c}2.04 \mathrm{~b} \pm 0.04 \\
(29.78)\end{array}$ & $\begin{array}{c}2.11 b \pm 0.10 \\
(31.10)\end{array}$ & $\begin{array}{c}1.01 \mathrm{a} \pm 0.03 \\
(13.53)\end{array}$ & $\begin{array}{c}0.86 \mathrm{a} \pm 0.22 \\
(25.55)\end{array}$ & $\begin{array}{c}0.82 \mathrm{a} \pm 0.07 \\
(26.54)\end{array}$ & $\begin{array}{l}0.96 \mathrm{a} \pm 0.07 \\
\quad(26.45)\end{array}$ \\
\hline & 40 & $\begin{array}{c}2.42 \mathrm{a} \pm 0.03 \\
(20.20)\end{array}$ & $\begin{array}{c}1.63 b \pm 0.04 \\
(46.49)\end{array}$ & $\begin{array}{c}1.57 \mathrm{~b} \pm 0.03 \\
\quad(45.90)\end{array}$ & $\begin{array}{c}1.64 b \pm 0.10 \\
(46.54)\end{array}$ & $\begin{array}{c}0.95 \mathrm{a} \pm 0.07 \\
\quad(18.29)\end{array}$ & $\begin{array}{c}0.67 \mathrm{~b} \pm 0.14 \\
\quad(42.20)\end{array}$ & $\begin{array}{c}0.63 b \pm 0.07 \\
\quad(43.27)\end{array}$ & $\begin{array}{l}0.74 \mathrm{~b} \pm 0.06 \\
\quad(43.27)\end{array}$ \\
\hline & 60 & $\begin{array}{c}2.06 a \pm 0.07 \\
(31.98)\end{array}$ & $\begin{array}{l}0.78 \mathrm{~b} \pm 0.10 \\
\quad(74.32)\end{array}$ & $\begin{array}{c}0.77 \mathrm{~b} \pm 0.03 \\
\quad(73.53)\end{array}$ & $\begin{array}{c}0.79 \mathrm{~b} \pm 0.04 \\
(74.09)\end{array}$ & $\begin{array}{c}0.82 \mathrm{a} \pm 0.13 \\
(29.56)\end{array}$ & $\begin{array}{c}0.38 \mathrm{~b} \pm 0.03 \\
(67.50)\end{array}$ & $\begin{array}{l}0.34 \mathrm{~b} \pm 0.05 \\
\quad(68.95)\end{array}$ & $\begin{array}{l}0.39 \mathrm{~b} \pm 0.06 \\
\quad(70.44)\end{array}$ \\
\hline \multirow{4}{*}{ Weedy rice } & 0 & $\begin{array}{c}3.89 \mathrm{a} \pm 0.08 \\
(0)\end{array}$ & $\begin{array}{c}3.86 a \pm 0.10 \\
(0)\end{array}$ & $\begin{array}{c}3.90 \mathrm{a} \pm 0.16 \\
(0)\end{array}$ & $\begin{array}{c}3.95 a \pm 0.12 \\
(0)\end{array}$ & $\begin{array}{c}2.37 \mathrm{a} \pm 0.17 \\
(0)\end{array}$ & $\begin{array}{c}2.23 a \pm 0.17 \\
(0)\end{array}$ & $\begin{array}{c}2.12 \mathrm{a} \pm 0.22 \\
(0)\end{array}$ & $\begin{array}{c}2.15 \mathrm{a} \pm 0.20 \\
(0)\end{array}$ \\
\hline & 20 & $\begin{array}{c}3.80 \mathrm{a} \pm 0.08 \\
(2.33)\end{array}$ & $\begin{array}{c}3.45 \mathrm{~b} \pm 0.07 \\
(10.61)\end{array}$ & $\begin{array}{c}3.37 \mathrm{c} \pm 0.10 \\
(13.59)\end{array}$ & $\begin{array}{c}3.18 \mathrm{c} \pm 0.03 \\
(19.34)\end{array}$ & $\begin{array}{c}2.32 \mathrm{a} \pm 0.02 \\
(2.01)\end{array}$ & $\begin{array}{c}2.01 b \pm 0.08 \\
(9.74)\end{array}$ & $\begin{array}{c}1.94 \mathrm{~b} \pm 0.20 \\
(8.30)\end{array}$ & $\begin{array}{c}1.92 \mathrm{~b} \pm 0.13 \\
\quad(10.75)\end{array}$ \\
\hline & 40 & $\begin{array}{c}3.59 \mathrm{a} \pm 0.04 \\
(7.70)\end{array}$ & $\begin{array}{c}3.06 \mathrm{~b} \pm 0.10 \\
(20.86)\end{array}$ & $\begin{array}{c}3.11 b \pm 0.09 \\
(20.37)\end{array}$ & $\begin{array}{c}3.10 \mathrm{~b} \pm 0.10 \\
(21.41)\end{array}$ & $\begin{array}{c}2.20 \mathrm{a} \pm 0.16 \\
(6.97)\end{array}$ & $\begin{array}{c}1.85 \mathrm{~b} \pm 0.09 \\
(16.87)\end{array}$ & $\begin{array}{c}1.75 b \pm 0.11 \\
(17.51)\end{array}$ & $\begin{array}{l}1.75 \mathrm{~b} \pm 0.11 \\
\quad(17.93)\end{array}$ \\
\hline & 60 & $\begin{array}{c}3.39 \mathrm{a} \pm 0.03 \\
(12.80)\end{array}$ & $\begin{array}{l}2.56 \mathrm{~b} \pm 0.06 \\
(33.75)\end{array}$ & $\begin{array}{c}2.59 \mathrm{~b} \pm 0.05 \\
\quad(33.73)\end{array}$ & $\begin{array}{l}2.52 b \pm 0.05 \\
(36.21)\end{array}$ & $\begin{array}{c}2.11 \mathrm{a} \pm 0.09 \\
(10.96)\end{array}$ & $\begin{array}{c}1.51 \mathrm{~b} \pm 0.06 \\
(32.08)\end{array}$ & $\begin{array}{c}1.43 \mathrm{~b} \pm 0.09 \\
(32.23)\end{array}$ & $\begin{array}{c}1.47 \mathrm{~b} \pm 0.07 \\
\quad(31.68)\end{array}$ \\
\hline \multirow{4}{*}{ C. iria } & 0 & $\begin{array}{c}3.90 \mathrm{ab} \pm \\
0.07(0)\end{array}$ & $\begin{array}{c}3.78 \mathrm{~b} \pm 0.05 \\
(0)\end{array}$ & $\begin{array}{c}3.86 \mathrm{ab} \pm 0.09 \\
(0)\end{array}$ & $\begin{array}{c}3.99 \mathrm{a} \pm 0.05 \\
(0)\end{array}$ & $\begin{array}{c}3.39 \mathrm{a} \pm 0.09 \\
(0)\end{array}$ & $\begin{array}{c}3.07 \mathrm{bc} \pm 0.12 \\
(0)\end{array}$ & $\begin{array}{c}3.29 \mathrm{ab} \pm 0.10 \\
(0)\end{array}$ & $\begin{array}{c}2.83 c \pm 0.13 \\
(0)\end{array}$ \\
\hline & 20 & $\begin{array}{c}3.75 a \pm 0.04 \\
(3.63)\end{array}$ & $\begin{array}{c}3.05 \mathrm{~b} \pm 0.11 \\
(19.30)\end{array}$ & $\begin{array}{c}3.10 \mathrm{~b} \pm 0.07 \\
(19.65)\end{array}$ & $\begin{array}{c}3.19 b \pm 0.07 \\
(20.12)\end{array}$ & $\begin{array}{c}3.28 \mathrm{a} \pm 0.06 \\
(3.36)\end{array}$ & $\begin{array}{c}2.53 \mathrm{~b} \pm 0.29 \\
(17.60)\end{array}$ & $\begin{array}{c}2.68 \mathrm{~b} \pm 0.28 \\
(18.53)\end{array}$ & $\begin{array}{c}2.30 \mathrm{~b} \pm 0.06 \\
\quad(18.71)\end{array}$ \\
\hline & 40 & $\begin{array}{c}3.54 a \pm 0.06 \\
(9.02)\end{array}$ & $\begin{array}{c}2.79 b \pm 0.07 \\
(26.09)\end{array}$ & $\begin{array}{c}2.85 b \pm 0.07 \\
(26.05)\end{array}$ & $\begin{array}{c}2.84 b \pm 0.05 \\
(28.76)\end{array}$ & $\begin{array}{c}3.13 \mathrm{a} \pm 0.06 \\
(7.68)\end{array}$ & $\begin{array}{c}2.39 b \pm 0.07 \\
(22.24)\end{array}$ & $\begin{array}{c}2.51 b c \pm 0.16 \\
(23.75)\end{array}$ & $\begin{array}{l}2.15 \mathrm{c} \pm 0.10 \\
\quad(22.61)\end{array}$ \\
\hline & 60 & $\begin{array}{c}3.27 \mathrm{a} \pm 0.05 \\
(16.15)\end{array}$ & $\begin{array}{c}2.42 \mathrm{c} \pm 0.03 \\
(36.03)\end{array}$ & $\begin{array}{c}2.45 \mathrm{bc} \pm 0.06 \\
\quad(36.35)\end{array}$ & $\begin{array}{c}2.54 b \pm 0.06 \\
(36.31)\end{array}$ & $\begin{array}{c}2.99 a \pm 0.01 \\
(11.72)\end{array}$ & $\begin{array}{c}2.00 \mathrm{c} \pm 0.07 \\
(34.88)\end{array}$ & $\begin{array}{c}2.24 \mathrm{~b} \pm 0.06 \\
(31.86)\end{array}$ & $\begin{array}{c}1.93 \mathrm{c} \pm 0.05 \\
(32.00)\end{array}$ \\
\hline
\end{tabular}

Data are expressed as means \pm SD. Values with the same letters in the row among the hours after spray for each test weed are not significantly different at $p>0.05$. Values inside the parenthesis are inhibition percentages relative to the control.

\subsection{Effect of P. hysterophorus Extract on Chlorophyll-b Content of the Test Plant Species}

The chlorophyll- $b$ content was significantly affected by the foliar spray of $P$. hysterophorus extract, but no significant effect was observed at $20 \mathrm{~g} \mathrm{~L}^{-1}$ between the HAS in A. conyzoides (Table 1). The foliar spray concentration, however, reduced the chlorophyll- $b$ content of $A$. conyzoides at 24 HAS from 25.55 to $67.50 \%$ at the lowest $\left(20 \mathrm{~g} \mathrm{~L}^{-1}\right)$ to highest $\left(60 \mathrm{~g} \mathrm{~L}^{-1}\right)$ concentration compared to the control $\left(0 \mathrm{~g} \mathrm{~L}^{-1}\right)$. The result also exhibited that the response of weedy rice was not significantly different in the lowest $\left(20 \mathrm{~g} \mathrm{~L}^{-1}\right)$ concentration but differed from the highest $\left(60 \mathrm{~g} \mathrm{~L}^{-1}\right)$ concentration and control $\left(0 \mathrm{~g} \mathrm{~L}^{-1}\right)$ at different hours of spray. C. iria showed a $34.88 \%$ decreased in chlorophyll- $b$ content compared to control $\left(0 \mathrm{~g} \mathrm{~L}^{-1}\right)$ at 24 HAS at the highest $\left(60 \mathrm{~g} \mathrm{~L}^{-1}\right)$ concentration of $P$. hysterophorus extract. $A$. conyzoides showed the highest reduction in chlorophyll- $b$ content compared to other test plants and this was a relatively much greater impact on the green pigment.

\subsection{Effect of P. hysterophorus Extract on Total Chlorophyll Content of the Test Plant Species}

Total chlorophyll content was significantly affected by the interaction of hours after foliar spray and P. hysterophorus extract concentration. The total chlorophyll content of A. conyzoides was declined $12.79 \%, 19.67 \%$, and $31.31 \%$ at 6 HAS, while $22.78 \%, 45.31 \%$ and $72.45 \%$ reduction was obtained at $24 \mathrm{HAS}$ for the foliar application of $20 \mathrm{~g} \mathrm{~L}^{-1}$, $40 \mathrm{~g} \mathrm{~L}^{-1}$ and $60 \mathrm{~g} \mathrm{~L}^{-1}$, respectively compared to control $\left(0 \mathrm{~g} \mathrm{~L}^{-1}\right)$ (Table 2$)$. Around $34 \%$ reduction occurred in the total chlorophyll content of weedy rice, and C. iria at 72 HAS by applying the highest $\left(60 \mathrm{~g} \mathrm{~L}^{-1}\right)$ amount of $P$. hysterophorus extract. Weedy rice and $C$. iria maintained relatively higher chlorophyll content than $A$. conyzoides, and it is also revealed that having a higher amount of chlorophyll- $a$ and chlorophyll- $b$ is an indication of higher total chlorophyll content. 
Table 2. Response of total chlorophyll and carotenoids content of A. conyzoides, weedy rice and C. iria to a foliar spray of P. hysterophorus extract.

\begin{tabular}{|c|c|c|c|c|c|c|c|c|c|}
\hline \multirow{3}{*}{ Test Plants } & \multirow{3}{*}{$\begin{array}{c}\text { Dose } \\
\left(\mathrm{g} \mathrm{L}^{-1}\right)\end{array}$} & \multicolumn{4}{|c|}{ Total Chlorophyll (mg g ${ }^{-1}$ FW) } & \multicolumn{4}{|c|}{ Carotenoids (mg g $\left.{ }^{-1} \mathrm{FW}\right)$} \\
\hline & & \multicolumn{4}{|c|}{ Hours after Spray } & \multicolumn{4}{|c|}{ Hours after Spray } \\
\hline & & 6 & 24 & 48 & 72 & 6 & 24 & 48 & 72 \\
\hline \multirow{4}{*}{$\begin{array}{c}\text { A. } \\
\text { conyzoides }\end{array}$} & 0 & $\begin{array}{c}4.17 \mathrm{ab} \pm \\
0.05(0)\end{array}$ & $\begin{array}{c}4.20 \mathrm{ab} \pm 0.04 \\
(0)\end{array}$ & $\begin{array}{c}4.02 b \pm 0.09 \\
(0)\end{array}$ & $\begin{array}{c}4.37 \mathrm{a} \pm 0.08 \\
(0)\end{array}$ & $\begin{array}{c}1.02 b \pm 0.03 \\
(0)\end{array}$ & $\begin{array}{c}1.03 a \pm 0.03 \\
(0)\end{array}$ & $\begin{array}{c}1.01 \mathrm{a} \pm 0.02 \\
(0)\end{array}$ & $\begin{array}{c}1.14 \mathrm{a} \pm 0.03 \\
(0)\end{array}$ \\
\hline & 20 & $\begin{array}{c}3.66 \mathrm{a} \pm 0.15 \\
(12.79)\end{array}$ & $\begin{array}{c}2.99 \mathrm{~b} \pm 0.18 \\
(22.78)\end{array}$ & $\begin{array}{c}2.86 \mathrm{~b} \pm 0.08 \\
(28.88)\end{array}$ & $\begin{array}{c}3.07 \mathrm{~b} \pm 0.08 \\
(29.70)\end{array}$ & $\begin{array}{c}0.86 \mathrm{a} \pm 0.03 \\
\quad(16.82)\end{array}$ & $\begin{array}{c}0.70 \mathrm{c} \pm 0.02 \\
(31.94)\end{array}$ & $\begin{array}{c}0.68 c \pm 0.03 \\
(32.30)\end{array}$ & $\begin{array}{l}0.77 \mathrm{~b} \pm 0.01 \\
\quad(32.08)\end{array}$ \\
\hline & 40 & $\begin{array}{c}3.37 \mathrm{a} \pm 0.10 \\
(19.67)\end{array}$ & $\begin{array}{c}2.30 \mathrm{~b} \pm 0.11 \\
(45.31)\end{array}$ & $\begin{array}{c}2.20 \mathrm{~b} \pm 0.07 \\
(45.17)\end{array}$ & $\begin{array}{c}2.38 \mathrm{~b} \pm 0.10 \\
\quad(45.56)\end{array}$ & $\begin{array}{c}0.81 \mathrm{a} \pm 0.02 \\
(21.01)\end{array}$ & $\begin{array}{c}0.55 c \pm 0.01 \\
(46.67)\end{array}$ & $\begin{array}{c}0.54 \mathrm{c} \pm 0.01 \\
(46.71)\end{array}$ & $\begin{array}{c}0.60 \mathrm{~b} \pm 0.01 \\
\quad(47.04)\end{array}$ \\
\hline & 60 & $\begin{array}{c}2.88 \mathrm{a} \pm 0.16 \\
(31.31)\end{array}$ & $\begin{array}{c}1.16 \mathrm{~b} \pm 0.11 \\
(72.45)\end{array}$ & $\begin{array}{c}1.11 \mathrm{~b} \pm 0.07 \\
(72.26)\end{array}$ & $\begin{array}{c}1.18 \mathrm{~b} \pm 0.06 \\
(73.00)\end{array}$ & $\begin{array}{c}0.69 \mathrm{a} \pm 0.03 \\
(32.89)\end{array}$ & $\begin{array}{l}0.30 \mathrm{~b} \pm 0.01 \\
\quad(70.94)\end{array}$ & $\begin{array}{c}0.30 \mathrm{~b} \pm 0.01 \\
(70.46)\end{array}$ & $\begin{array}{l}0.33 \mathrm{~b} \pm 0.02 \\
\quad(71.29)\end{array}$ \\
\hline \multirow{4}{*}{ Weedy rice } & 0 & $\begin{array}{c}6.26 \mathrm{a} \pm 0.10 \\
(0)\end{array}$ & $\begin{array}{c}6.09 \mathrm{a} \pm 0.10 \\
(0)\end{array}$ & $\begin{array}{c}6.02 \mathrm{a} \pm 0.18 \\
(0)\end{array}$ & $\begin{array}{c}6.10 \mathrm{a} \pm 0.10 \\
(0)\end{array}$ & $\begin{array}{c}1.84 \mathrm{a} \pm 0.03 \\
(0)\end{array}$ & $\begin{array}{c}1.47 \mathrm{a} \pm 0.70 \\
(0)\end{array}$ & $\begin{array}{c}1.78 \mathrm{a} \pm 0.07 \\
(0)\end{array}$ & $\begin{array}{c}1.80 \mathrm{a} \pm 0.06 \\
(0)\end{array}$ \\
\hline & 20 & $\begin{array}{c}6.12 \mathrm{a} \pm 0.07 \\
(2.21)\end{array}$ & $\begin{array}{c}5.46 \mathrm{~b} \pm 0.06 \\
(10.29)\end{array}$ & $\begin{array}{c}5.31 \mathrm{bc} \pm 0.12 \\
(11.73)\end{array}$ & $\begin{array}{c}5.10 \mathrm{c} \pm 0.13 \\
(16.32)\end{array}$ & $\begin{array}{c}1.79 \mathrm{a} \pm 0.06 \\
(2.65)\end{array}$ & $\begin{array}{c}1.30 \mathrm{c} \pm 0.02 \\
(11.67)\end{array}$ & $\begin{array}{c}1.53 \mathrm{~b} \pm 0.03 \\
(13.64)\end{array}$ & $\begin{array}{c}1.49 \mathrm{~b} \pm 0.07 \\
\quad(17.05)\end{array}$ \\
\hline & 40 & $\begin{array}{c}5.79 \mathrm{a} \pm 0.14 \\
(7.43)\end{array}$ & $\begin{array}{c}4.91 \mathrm{~b} \pm 0.05 \\
(19.40)\end{array}$ & $\begin{array}{c}4.85 \mathrm{~b} \pm 0.15 \\
(19.36)\end{array}$ & $\begin{array}{c}4.87 \mathrm{~b} \pm 0.05 \\
(20.18)\end{array}$ & $\begin{array}{c}1.71 \mathrm{a} \pm 0.02 \\
(7.13)\end{array}$ & $\begin{array}{c}1.15 c \pm 0.03 \\
(21.89)\end{array}$ & $\begin{array}{c}1.40 \mathrm{~b} \pm 0.09 \\
(21.22)\end{array}$ & $\begin{array}{c}1.40 \mathrm{~b} \pm 0.01 \\
(22.16)\end{array}$ \\
\hline & 60 & $\begin{array}{c}5.50 \mathrm{a} \pm 0.09 \\
(12.10)\end{array}$ & $\begin{array}{c}4.07 \mathrm{~b} \pm 0.05 \\
(33.14)\end{array}$ & $\begin{array}{c}4.02 \mathrm{~b} \pm 0.04 \\
(32.20)\end{array}$ & $\begin{array}{c}3.99 \mathrm{~b} \pm 0.10 \\
(34.62)\end{array}$ & $\begin{array}{c}1.64 \mathrm{a} \pm 0.02 \\
(10.82)\end{array}$ & $\begin{array}{c}1.03 c \pm 0.01 \\
(30.08)\end{array}$ & $\begin{array}{c}1.24 \mathrm{~b} \pm 0.04 \\
(30.01)\end{array}$ & $\begin{array}{c}1.21 \mathrm{~b} \pm 0.02 \\
\quad(32.45)\end{array}$ \\
\hline \multirow{4}{*}{ C. iria } & 0 & $\begin{array}{c}7.29 \mathrm{a} \pm 0.06 \\
(0)\end{array}$ & $\begin{array}{c}6.85 b \pm 0.10 \\
(0)\end{array}$ & $\begin{array}{c}7.15 a \pm 0.04 \\
(0)\end{array}$ & $\begin{array}{c}6.82 \mathrm{~b} \pm 0.12 \\
(0)\end{array}$ & $\begin{array}{c}1.68 \mathrm{~b} \pm 0.03 \\
(0)\end{array}$ & $\begin{array}{c}1.73 b \pm 0.03 \\
(0)\end{array}$ & $\begin{array}{c}1.81 \mathrm{a} \pm 0.03 \\
(0)\end{array}$ & $\begin{array}{c}1.80 \mathrm{a} \pm 0.04 \\
(0)\end{array}$ \\
\hline & 20 & $\begin{array}{c}7.03 \mathrm{a} \pm 0.07 \\
(3.51)\end{array}$ & $\begin{array}{c}5.58 \mathrm{~b} \pm 0.24 \\
(18.54)\end{array}$ & $\begin{array}{c}5.78 b \pm 0.22 \\
\quad(19.14)\end{array}$ & $\begin{array}{c}5.49 \mathrm{~b} \pm 0.11 \\
(19.53)\end{array}$ & $\begin{array}{c}1.61 \mathrm{a} \pm 0.03 \\
(3.84)\end{array}$ & $\begin{array}{c}1.48 \mathrm{a} \pm 0.06 \\
(14.33)\end{array}$ & $\begin{array}{l}1.48 \mathrm{a} \pm 0.10 \\
(18.43)\end{array}$ & $\begin{array}{c}1.48 \mathrm{a} \pm 0.06 \\
\quad(18.06)\end{array}$ \\
\hline & 40 & $\begin{array}{c}6.67 \mathrm{a} \pm 0.1 \\
(8.40)\end{array}$ & $\begin{array}{c}5.18 \mathrm{bc} \pm 0.06 \\
(24.37)\end{array}$ & $\begin{array}{c}5.36 \mathrm{~b} \pm 0.11 \\
(24.99)\end{array}$ & $\begin{array}{c}5.00 \mathrm{c} \pm 0.14 \\
(26.21)\end{array}$ & $\begin{array}{c}1.54 \mathrm{a} \pm 0.02 \\
(8.05)\end{array}$ & $\begin{array}{c}1.26 \mathrm{~b} \pm 0.02 \\
(27.36)\end{array}$ & $\begin{array}{c}1.32 \mathrm{~b} \pm 0.03 \\
(27.43)\end{array}$ & $\begin{array}{c}1.29 \mathrm{~b} \pm 0.15 \\
(28.32)\end{array}$ \\
\hline & 60 & $\begin{array}{c}6.26 \mathrm{a} \pm 0.03 \\
(14.09)\end{array}$ & $\begin{array}{c}4.42 \mathrm{c} \pm 0.06 \\
(35.51)\end{array}$ & $\begin{array}{c}4.70 \mathrm{~b} \pm 0.05 \\
(34.28)\end{array}$ & $\begin{array}{c}4.47 \mathrm{c} \pm 0.06 \\
(34.52)\end{array}$ & $\begin{array}{c}1.49 \mathrm{a} \pm 0.01 \\
(11.41)\end{array}$ & $\begin{array}{c}1.14 \mathrm{~b} \pm 0.03 \\
(34.19)\end{array}$ & $\begin{array}{c}1.20 \mathrm{~b} \pm 0.03 \\
(34.07)\end{array}$ & $\begin{array}{c}1.17 \mathrm{~b} \pm 0.04 \\
\quad(35.32)\end{array}$ \\
\hline
\end{tabular}

Data are expressed as mean \pm SD. Values with the same letters in the row among the hours after spray for each test weed are not significantly different at $p>0.05$. Values inside the parenthesis are inhibition percentages relative to the control.

\subsection{Effect of P. hysterophorus Extract on Carotenoid Content of the Test Plant Species}

Carotenoid contents of $A$. conyzoides, weedy rice and C. iria were significantly affected by the foliar application of P. hysterophorus extract at different exposure times (Table 2). At 6 HAS P. hysterophorus extract reduced the carotenoid contents of $A$. conyzoides, weedy rice and C. iria from 16.82 to $32.89 \%, 2.65$ to $10.82 \%$ and 3.84 to $11.41 \%$, respectively at lowest $\left(20 \mathrm{~g} \mathrm{~L}^{-1}\right)$ to highest $\left(60 \mathrm{~g} \mathrm{~L}^{-1}\right)$ concentration compared to the control $\left(0 \mathrm{~g} \mathrm{~L}^{-1}\right)$. The highest inhibition was started to occur for all test plants at $24 \mathrm{HAS}$, while the lowest carotenoid content was observed in A. conyzoides compared to weedy rice and C. iria. Carotenoid contents showed a decline of $71.29 \%, 32.45 \%$ and $35.32 \%$ in $A$. conyzoides, weedy rice and C. iria, respectively, at 72 HAS in the presence of the highest concentration of P. hysterophorus.

\subsection{Effect of P. hysterophorus Extract on Photosynthesis Rate of the Test Plant Species}

The significant interaction effect of time after spray and extract concentration was observed on the photosynthesis rate of $A$. conyzoides, weedy rice and C. iria (Table 3 ). The different exposure times of $P$. hysterophorus extract significantly decreased the rate of photosynthesis of test plants. The extract levels had triggered different responses by the species, and this could be attributed to the inherent genetic variability among the species. Nevertheless, A. conyzoides had expressed more reduction in photosynthetic rate across the species with inhibition of $21.73 \%$, while $7.82 \%$ and $10.54 \%$ were observed in weedy rice and C. iria, respectively at $6 \mathrm{HAS}$ with the highest $\left(60 \mathrm{~g} \mathrm{~L}^{-1}\right)$ concentration of P. hysterophorus. Among the times of spray, photosynthesis rate was more reduced at $72 \mathrm{HAS}$ in $A$. conyzoides at higher $\left(60 \mathrm{~g} \mathrm{~L}^{-1}\right)$ concentration of extract with an inhibition index of $78.85 \%$, followed by $36.33 \%$ and $28.37 \%$ in C. iria and weedy rice, respectively. 
Table 3. Response of photosynthesis rate, stomatal conductance and transpiration rate of $A$. conyzoides, weedy rice and $C$. iria to a foliar spray of $P$. hysterophorus extract.

\begin{tabular}{|c|c|c|c|c|c|c|c|c|c|c|c|c|c|}
\hline \multirow{3}{*}{ Test Plants } & \multirow{3}{*}{$\begin{array}{c}\text { Dose } \\
\left(\mathrm{g} \mathrm{L}^{-1}\right)\end{array}$} & \multicolumn{4}{|c|}{ Photosynthesis Rate $\left(\mu \mathrm{mol} \mathrm{m} \mathrm{m}^{-2} \mathrm{~s}^{-1}\right)$} & \multicolumn{4}{|c|}{ Stomatal Conductance $\left(\mathrm{mol} \mathrm{m}^{-2} \mathrm{~s}^{-1}\right)$} & \multicolumn{4}{|c|}{ Transpiration Rate $\left(\mathrm{mmol} \mathrm{m}^{-2} \mathrm{~s}^{-1}\right)$} \\
\hline & & \multirow{2}{*}{\multicolumn{3}{|c|}{$\begin{array}{rr}\text { Hours after Spray } \\
24\end{array}$}} & \multirow[b]{2}{*}{72} & \multicolumn{4}{|c|}{ Hours after Spray } & \multicolumn{4}{|c|}{ Hours after Spray } \\
\hline & & & & & & 6 & 24 & 48 & 72 & 6 & 24 & 48 & 72 \\
\hline \multirow{4}{*}{$\begin{array}{c}\text { A. } \\
\text { conyzoides }\end{array}$} & 0 & $\begin{array}{c}16.06 \mathrm{a} \pm \\
0.06(0)\end{array}$ & $\begin{array}{c}14.34 c \pm 0.03 \\
\text { (0) }\end{array}$ & $\begin{array}{l}\text { 13.70d } \pm 0.01 \\
(0)\end{array}$ & $\begin{array}{c}14.60 \mathrm{~b} \pm 0.05 \\
(0)\end{array}$ & $\begin{array}{l}0.41 \mathrm{a} \pm 0.01 \\
(0)\end{array}$ & $\begin{array}{c}0.35 c \pm 0.02 \\
\text { (0) }\end{array}$ & $\begin{array}{c}0.39 \mathrm{~b} \pm 0.01 \\
(0)\end{array}$ & $\begin{array}{c}0.40 \mathrm{~b} \pm 0.03 \\
(0)\end{array}$ & $\begin{array}{l}7.22 \mathrm{a} \pm 0.04 \\
(0)\end{array}$ & $\begin{array}{c}6.00 \mathrm{~b} \pm 0.01 \\
(0)\end{array}$ & $\begin{array}{c}5.03 c \pm 0.01 \\
(0)\end{array}$ & $\begin{array}{c}5.00 c \pm 0.06 \\
(0)\end{array}$ \\
\hline & 20 & $\begin{array}{c}15.00 \mathrm{a} \pm \\
0.39(6.56)\end{array}$ & $\begin{array}{c}10.35 b \pm 1.25 \\
(27.86)\end{array}$ & $\begin{array}{c}10.15 b \pm 0.03 \\
(25.89)\end{array}$ & $\begin{array}{c}10.40 \mathrm{~b} \pm 0.32 \\
(28.79)\end{array}$ & $\begin{array}{l}0.39 \mathrm{a} \pm 0.01 \\
\quad(4.40)\end{array}$ & $\begin{array}{l}0.29 \mathrm{~b} \pm 0.06 \\
\quad(17.94)\end{array}$ & $\begin{array}{c}0.30 \mathrm{~b} \pm 0.02 \\
\quad(23.61)\end{array}$ & $\begin{array}{c}0.30 \mathrm{~b} \pm 0.05 \\
\quad(23.59)\end{array}$ & $\begin{array}{c}6.80 \mathrm{a} \pm 0.11 \\
(5.84)\end{array}$ & $\begin{array}{c}4.19 b \pm 0.33 \\
\quad(30.20)\end{array}$ & $\begin{array}{c}3.80 \mathrm{~b} \pm 0.12 \\
\quad(24.33)\end{array}$ & $\begin{array}{c}3.73 \mathrm{~b} \pm 0.23 \\
\quad(25.28)\end{array}$ \\
\hline & 40 & $\begin{array}{c}14.32 \mathrm{a} \pm \\
0.44(10.83)\end{array}$ & $\begin{array}{c}8.10 \mathrm{~b} \pm 0.63 \\
(43.54)\end{array}$ & $\begin{array}{c}6.76 c \pm 0.01 \\
(60.61)\end{array}$ & $\begin{array}{c}7.29 \mathrm{bc} \pm 0.14 \\
(50.09)\end{array}$ & $\begin{array}{c}0.38 \mathrm{a} \pm 0.03 \\
\quad(7.50)\end{array}$ & $\begin{array}{c}0.21 \mathrm{~b} \pm 0.01 \\
\quad(39.15)\end{array}$ & $\begin{array}{l}0.20 \mathrm{~b} \pm 0.05 \\
\quad(48.42)\end{array}$ & $\begin{array}{c}0.21 \mathrm{~b} \pm 0.02 \\
\quad(47.54)\end{array}$ & $\begin{array}{c}6.55 \mathrm{a} \pm 0.01 \\
(9.27)\end{array}$ & $\begin{array}{l}2.75 b \pm 0.54 \\
\quad(54.08)\end{array}$ & $\begin{array}{l}2.26 \mathrm{~b} \pm 0.11 \\
\quad(55.00)\end{array}$ & $\begin{array}{c}2.24 \mathrm{~b} \pm 0.17 \\
\quad(55.09)\end{array}$ \\
\hline & 60 & $\begin{array}{c}12.57 \mathrm{a} \pm \\
0.06(21.73)\end{array}$ & $\begin{array}{c}3.93 \mathrm{~b} \pm 0.19 \\
(72.61)\end{array}$ & $\begin{array}{c}3.34 \mathrm{c} \pm 0.44 \\
(75.62)\end{array}$ & $\begin{array}{c}3.09 \mathrm{c} \pm 0.12 \\
(78.85)\end{array}$ & $\begin{array}{c}0.32 \mathrm{a} \pm 0.02 \\
(20.38)\end{array}$ & $\begin{array}{c}0.14 \mathrm{bc} \pm \\
0.01(59.54)\end{array}$ & $\begin{array}{c}0.15 b \pm 0.07 \\
\quad(62.36)\end{array}$ & $\begin{array}{c}0.12 c \pm 0.05 \\
\quad(69.37)\end{array}$ & $\begin{array}{c}5.73 a \pm 0.14 \\
(20.66)\end{array}$ & $\begin{array}{c}1.27 \mathrm{~b} \pm 0.07 \\
(78.81)\end{array}$ & $\begin{array}{c}1.09 \mathrm{bc} \pm \\
0.06(78.22)\end{array}$ & $\begin{array}{c}1.05 \mathrm{c} \pm 0.01 \\
(78.94)\end{array}$ \\
\hline \multirow{4}{*}{ Weedy rice } & 0 & $\begin{array}{c}48.71 \mathrm{a} \pm \\
0.29(0)\end{array}$ & $\begin{array}{c}45.53 b \pm 0.29 \\
(0)\end{array}$ & $\begin{array}{c}45.95 b \pm 0.68 \\
(0)\end{array}$ & $\begin{array}{c}45.45 b \pm 0.04 \\
(0)\end{array}$ & $\begin{array}{c}0.53 a \pm 0.01 \\
(0)\end{array}$ & $\begin{array}{c}0.58 \mathrm{ab} \pm \\
0.02(0)\end{array}$ & $\begin{array}{c}0.57 \mathrm{~b} \pm 0.03 \\
(0)\end{array}$ & $\begin{array}{l}0.61 \mathrm{c} \pm 0.04 \\
\quad(0)\end{array}$ & $\begin{array}{c}14.80 \mathrm{a} \pm \\
0.02(0)\end{array}$ & $\begin{array}{c}13.47 \mathrm{bc} \pm \\
0.01(0)\end{array}$ & $\begin{array}{c}13.17 \mathrm{c} \pm \\
0.05(0)\end{array}$ & $\begin{array}{c}14.08 \mathrm{~b} \pm 0.58 \\
(0)\end{array}$ \\
\hline & 20 & $\begin{array}{c}47.88 \mathrm{a} \pm \\
0.12(1.70)\end{array}$ & $\begin{array}{c}43.23 \mathrm{~b} \pm 0.52 \\
(5.04)\end{array}$ & $\begin{array}{c}43.33 b \pm 0.42 \\
\quad(5.70)\end{array}$ & $\begin{array}{c}42.67 \mathrm{~b} \pm 0.06 \\
(6.11)\end{array}$ & $\begin{array}{l}0.52 \mathrm{~b} \pm 0.01 \\
\quad(1.57)\end{array}$ & $\begin{array}{c}0.56 \mathrm{~b} \pm 0.02 \\
\quad(4.72)\end{array}$ & $\begin{array}{c}0.54 \mathrm{ab} \pm 1 \\
(4.92)\end{array}$ & $\begin{array}{l}0.57 a \pm 0.02 \\
\quad(6.36)\end{array}$ & $\begin{array}{c}14.65 \mathrm{a} \pm \\
0.16(1.02)\end{array}$ & $\begin{array}{c}12.35 \mathrm{~b} \pm \\
0.02(8.37)\end{array}$ & $\begin{array}{c}12.10 \mathrm{~b} \pm \\
0.10(8.14)\end{array}$ & $\begin{array}{c}12.78 \mathrm{~b} \pm 0.91 \\
\quad(9.22)\end{array}$ \\
\hline & 40 & $\begin{array}{c}46.78 \mathrm{a} \pm \\
0.20(3.93)\end{array}$ & $\begin{array}{c}40.95 b \pm 0.13 \\
\quad(10.05)\end{array}$ & $\begin{array}{c}39.07 \mathrm{c} \pm 0.11 \\
(14.96)\end{array}$ & $\begin{array}{c}38.10 \mathrm{~d} \pm 0.05 \\
\quad(16.16)\end{array}$ & $\begin{array}{l}0.51 \mathrm{~b} \pm 0.01 \\
\quad(3.29)\end{array}$ & $\begin{array}{l}0.54 a \pm 0.01 \\
\quad(7.03)\end{array}$ & $\begin{array}{c}0.51 \mathrm{~b} \pm 0.02 \\
\quad(9.97)\end{array}$ & $\begin{array}{c}0.55 a \pm 0.05 \\
\quad(9.03)\end{array}$ & $\begin{array}{c}14.23 \mathrm{a} \pm \\
0.01(3.84)\end{array}$ & $\begin{array}{c}11.36 \mathrm{~b} \pm \\
0.02(15.79)\end{array}$ & $\begin{array}{c}11.20 \mathrm{~b} \pm \\
0.09(15.00)\end{array}$ & $\begin{array}{c}11.42 \mathrm{~b} \pm 1.26 \\
(18.90)\end{array}$ \\
\hline & 60 & $\begin{array}{c}44.89 \mathrm{a} \pm \\
0.23(7.82)\end{array}$ & $\begin{array}{c}32.84 \mathrm{~b} \pm 0.10 \\
\quad(27.87)\end{array}$ & $\begin{array}{c}33.08 \mathrm{~b} \pm 0.69 \\
(28.00)\end{array}$ & $\begin{array}{c}32.56 \mathrm{~b} \pm 0.50 \\
(28.37)\end{array}$ & $\begin{array}{c}0.49 \mathrm{a} \pm 0.04 \\
\quad(6.96)\end{array}$ & $\begin{array}{l}0.45 \mathrm{~b} \pm 0.01 \\
\quad(22.20)\end{array}$ & $\begin{array}{c}0.44 \mathrm{~b} \pm 0.05 \\
\quad(23.28)\end{array}$ & $\begin{array}{c}0.50 \mathrm{a} \pm 0.01 \\
\quad(18.07)\end{array}$ & $\begin{array}{c}13.50 \mathrm{a} \pm \\
0.50(6.96) \\
\end{array}$ & $\begin{array}{c}9.63 \mathrm{~b} \pm 0.48 \\
\quad(28.53)\end{array}$ & $\begin{array}{c}19.14 \mathrm{~b} \pm \\
0.25(30.63)\end{array}$ & $\begin{array}{c}9.67 \mathrm{~b} \pm 0.59 \\
(31.32)\end{array}$ \\
\hline \multirow{3}{*}{ C. iria } & 0 & $\begin{array}{c}46.84 \mathrm{a} \pm \\
0.54(0)\end{array}$ & $\begin{array}{c}42.35 b \pm 0.10 \\
\text { (0) }\end{array}$ & $\begin{array}{c}42.23 b \pm 1.40 \\
(0)\end{array}$ & $\begin{array}{c}43.14 \mathrm{~b} \pm 0.08 \\
(0)\end{array}$ & $\begin{array}{c}0.41 \mathrm{a} \pm 0.04 \\
(0)\end{array}$ & $\begin{array}{c}0.43 a \pm 0.04 \\
(0)\end{array}$ & $\begin{array}{c}0.38 \mathrm{a} \pm 0.01 \\
(0)\end{array}$ & $\begin{array}{c}0.40 \mathrm{a} \pm 0.02 \\
(0)\end{array}$ & $\begin{array}{c}13.09 \mathrm{a} \pm \\
0.14(0)\end{array}$ & $\begin{array}{c}11.22 \mathrm{~b} \pm \\
0.07(0)\end{array}$ & $\begin{array}{c}11.22 \mathrm{~b} \pm \\
0.07(0)\end{array}$ & $\begin{array}{c}11.29 \mathrm{~b} \pm 0.01 \\
\text { (0) }\end{array}$ \\
\hline & 40 & $\begin{array}{c}44.24 \mathrm{a} \pm \\
0.45(5.54)\end{array}$ & $\begin{array}{c}36.25 \mathrm{~b} \pm 0.70 \\
(14.41)\end{array}$ & $\begin{array}{c}34.75 c \pm 0.19 \\
(17.72)\end{array}$ & $\begin{array}{c}35.79 \mathrm{~b} \pm 0.44 \\
(17.04)\end{array}$ & $\begin{array}{c}0.39 \mathrm{a} \pm 0.04 \\
\quad(3.50)\end{array}$ & $\begin{array}{l}0.39 a \pm 0.01 \\
\quad(9.58)\end{array}$ & $\begin{array}{c}0.33 \mathrm{a} \pm 0.02 \\
(13.38)\end{array}$ & $\begin{array}{c}0.34 \mathrm{a} \pm 0.03 \\
(15.00)\end{array}$ & $\begin{array}{c}12.23 \mathrm{a} \pm \\
0.88(96.55)\end{array}$ & $\begin{array}{c}9.21 \mathrm{~b} \pm 0.50 \\
\quad(17.87)\end{array}$ & $\begin{array}{c}8.96 \mathrm{~b} \pm 0.46 \\
(20.08)\end{array}$ & $\begin{array}{l}8.99 \mathrm{~b} \pm 1.10 \\
\quad(20.37)\end{array}$ \\
\hline & 60 & $\begin{array}{c}41.90 \mathrm{a} \pm \\
0.54(10.54)\end{array}$ & $\begin{array}{c}28.23 \mathrm{~b} \pm 1.12 \\
(33.33)\end{array}$ & $\begin{array}{c}26.90 \mathrm{~b} \pm 0.10 \\
(36.31)\end{array}$ & $\begin{array}{c}27.47 \mathrm{~b} \pm 0.40 \\
(36.33)\end{array}$ & $\begin{array}{l}0.38 \mathrm{a} \pm 0.10 \\
\quad(6.34)\end{array}$ & $\begin{array}{l}0.32 \mathrm{a} \pm 0.02 \\
(25.45)\end{array}$ & $\begin{array}{l}0.28 \mathrm{a} \pm 0.03 \\
(27.70)\end{array}$ & $\begin{array}{l}0.29 \mathrm{a} \pm 0.01 \\
(28.94)\end{array}$ & $\begin{array}{c}11.62 \mathrm{~A} \pm \\
0.01(11.25)\end{array}$ & $\begin{array}{c}7.32 b \pm 0.22 \\
(34.69)\end{array}$ & $\begin{array}{l}7.04 \mathrm{~b} \pm 0.03 \\
(37.18)\end{array}$ & $\begin{array}{c}6.95 \mathrm{~b} \pm 0.48 \\
(38.48)\end{array}$ \\
\hline
\end{tabular}

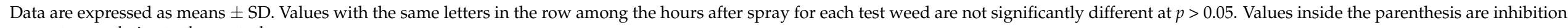

percentages relative to the control. 


\subsection{Effect of P. hysterophorus Extract on Stomatal Conductance of the Test Plant Species}

A significant effect was observed on stomatal conductance of $A$. conyzoides, weedy rice among different hours after spray (Table 3 ). The values of different exposure times in C. iria were not significantly different. There is a discernible difference in response to the extract concentration levels at a time after spray among the test species. Lower stomatal conductance was recorded in A. conyzoides $\left(0.35 \mathrm{~mol} \mathrm{~m}^{-2} \mathrm{~s}^{-1}\right)$, then C. iria $\left(0.38 \mathrm{~mol} \mathrm{~m}^{-2} \mathrm{~s}^{-1}\right)$ and weedy rice $\left(0.53 \mathrm{~mol} \mathrm{~m}^{-2} \mathrm{~s}^{-1}\right)$, and this indicated a remarkable variation in the integral phenomena of the test plant species. However, a minimum inhibition index of only $6.34 \%$ and $6.96 \%$ appeared in C. iria and weedy rice, respectively, while $A$. conyzoides was possessed the highest $(20.38 \%)$ inhibition at 6 HAS at the highest $\left(60 \mathrm{~g} \mathrm{~L}^{-1}\right)$ concentration. The highest inhibition was started at the highest $\left(60 \mathrm{~g} \mathrm{~L}^{-1}\right)$ concentration of P. hysterophorus extract at $24 \mathrm{HAS}$ with an inhibition index of $59.54 \%, 22.20 \%$ and $25.45 \%$ in A. conyzoides weedy rice and C. iria, respectively.

\subsection{Effect of P. hysterophorus Extract on Transpiration Rate of the Test Plant Species}

Foliar spray of $P$. hysterophorus extract had a significant effect on the transpiration rate of $A$. conyzoides, weedy rice and $C$. iria (Table 3). The P. hysterophorus extract at 20,40 , and $60 \mathrm{~g} \mathrm{~L}^{-1}$ concentrations resulted in $5.84 \%, 9.27 \%$ and $20.66 \%$ reduction at 6 HAS, while $25.28 \%, 55.09 \%$ and $78.94 \%$ reduction at 72 HAS in transpiration rate than control $\left(0 \mathrm{~g} \mathrm{~L}^{-1}\right)$, respectively for $A$. conyzoides. Weedy rice and $C$. iria were less sensitive to the extract concentration of $P$. hysterophorus compared to A. conyzoides. At $24 \mathrm{HAS}$, transpiration rate showed a reduction of $78.81 \%, 34.69 \%$ and $28.53 \%$ in A. conyzoides, C. iria and weedy rice, respectively, by the foliar application of the maximum $\left(60 \mathrm{~g} \mathrm{~L}^{-1}\right)$ concentration of $P$. hysterophorus. The lowest transpiration rate was observed for weedy rice $\left(9.67 \mathrm{mmol} \mathrm{H}_{2} \mathrm{O} \mathrm{m}^{-2} \mathrm{~s}^{-1}\right)$ and C. iria $\left(6.95 \mathrm{mmol} \mathrm{m}^{-2} \mathrm{~s}^{-1}\right)$ at $72 \mathrm{HAS}$ at the highest $\left(60 \mathrm{~g} \mathrm{~L}^{-1}\right)$ concentration, and the inhibition was $31.32 \%$ and $38.48 \%$, respectively.

\subsection{Effect of P. hysterophorus Extract on Malondialdehyde Content of the Test Plant Species}

Malondialdehyde (MDA) content was significantly lower in control for all the test plant species compared to the treated plants with the P. hysterophorus extract (Table 4). MDA content showed a concentration-dependent increase in response to $P$. hysterophorus extract. At 6 HAS, MDA content of A. conyzoides was increased by 21.99 to $83.70 \%$ at the lowest $\left(20 \mathrm{~g} \mathrm{~L}^{-1}\right)$ and highest $\left(60 \mathrm{~g} \mathrm{~L}^{-1}\right)$ concentrations, respectively compared to control $\left(0 \mathrm{~g} \mathrm{~L}^{-1}\right)$, and that for weedy rice and C. iria the values were 3.72 to $17.15 \%$ and 6.74 to $25.42 \%$, respectively (Figure 1). The MDA level of test weeds was unchanged after short exposure (i.e., 6 HAS) at the lowest concentration of $P$. hysterophorus. The MDA contents were significantly higher at long exposure time (24, 48 and 72 HAS) than short exposure time (6 HAS) to P. hysterophorus extract (Figure 1). The MDA levels of A. conyzoides were $155.66 \%$ higher than the control $\left(0 \mathrm{~g} \mathrm{~L}^{-1}\right)$ at $48 \mathrm{HAS}$ with the highest $\left(60 \mathrm{~g} \mathrm{~L}^{-1}\right)$ concentration of $P$. hysterophorus extract. 
Table 4. Response of malondialdehyde and proline content of $A$. conyzoides, weedy rice and C. iria to a foliar spray of $P$. hysterophorus extract.

\begin{tabular}{|c|c|c|c|c|c|c|c|c|c|}
\hline \multirow{3}{*}{ Test Plants } & \multirow{3}{*}{$\begin{array}{c}\text { Dose } \\
\left(\mathrm{g} \mathrm{L}^{-1}\right)\end{array}$} & \multicolumn{4}{|c|}{ Malondialdehyde Content $\left(\mu \mathrm{mol} \mathrm{g}{ }^{-1} \mathrm{FW}\right)$} & \multicolumn{4}{|c|}{ Proline Content $\left(\mu \mathrm{mol} \mathrm{g}^{-1} \mathrm{FW}\right)$} \\
\hline & & \multicolumn{4}{|c|}{ Hours after Spray } & \multicolumn{4}{|c|}{ Hours after Spray } \\
\hline & & 6 & 24 & 48 & 72 & 6 & 24 & 48 & 72 \\
\hline \multirow{4}{*}{ A. conyzoides } & 0 & $1.90 \mathrm{a} \pm 0.06$ & $1.79 \mathrm{a} \pm 0.14$ & $1.71 \mathrm{a} \pm 0.03$ & $1.79 \mathrm{a} \pm 0.14$ & $5.06 \mathrm{a} \pm 0.28$ & $4.93 a \pm 0.14$ & $5.01 \mathrm{a} \pm 0.15$ & $4.99 \mathrm{a} \pm 0.08$ \\
\hline & 20 & $2.32 \mathrm{a} \pm 0.09$ & $2.58 \mathrm{a} \pm 0.18$ & $2.45 a \pm 0.06$ & $2.62 a \pm 0.21$ & $6.60 \mathrm{~b} \pm 0.23$ & $7.03 a b \pm 0.14$ & $7.40 \mathrm{a} \pm 0.32$ & $7.12 \mathrm{a} \pm 0.10$ \\
\hline & 40 & $2.71 b \pm 0.08$ & $3.21 \mathrm{a} \pm 0.22$ & $3.14 \mathrm{a} \pm 0.03$ & $3.29 \mathrm{a} \pm 0.24$ & $7.83 b \pm 0.53$ & $9.12 \mathrm{a} \pm 0.29$ & $9.41 \mathrm{a} \pm 0.22$ & $9.37 \mathrm{a} \pm 0.06$ \\
\hline & 60 & $3.49 b \pm 0.06$ & $4.52 \mathrm{a} \pm 0.29$ & $4.37 \mathrm{a} \pm 0.05$ & $4.67 \mathrm{a} \pm 0.33$ & $9.57 \mathrm{~b} \pm 0.69$ & $12.14 \mathrm{a} \pm 0.42$ & $12.51 \mathrm{a} \pm 0.46$ & $12.58 \mathrm{a} \pm 0.08$ \\
\hline \multirow{4}{*}{ Weedy rice } & 0 & $2.80 \mathrm{a} \pm 0.11$ & $2.70 \mathrm{ab} \pm 0.06$ & $2.58 b \pm 0.09$ & $2.56 b \pm 0.11$ & $4.13 a \pm 0.24$ & $3.75 b \pm 0.20$ & $4.01 \mathrm{ab} \pm 0.07$ & $3.85 \mathrm{ab} \pm 0.14$ \\
\hline & 20 & $2.90 \mathrm{a} \pm 0.12$ & $3.12 \mathrm{a} \pm 0.11$ & $3.00 \mathrm{a} \pm 0.09$ & $2.98 \mathrm{a} \pm 0.12$ & $4.31 \mathrm{a} \pm 0.28$ & $4.29 a \pm 0.24$ & $4.67 a \pm 0.14$ & $4.51 \mathrm{a} \pm 0.28$ \\
\hline & 40 & $2.99 b \pm 0.08$ & $3.39 a \pm 0.15$ & $3.25 \mathrm{ab} \pm 0.12$ & $3.21 \mathrm{ab} \pm 0.19$ & $4.60 \mathrm{~b} \pm 0.21$ & $4.73 \mathrm{ab} \pm 0.28$ & $5.14 \mathrm{a} \pm 0.17$ & $5.01 \mathrm{ab} \pm 0.22$ \\
\hline & 60 & $3.28 b \pm 0.11$ & $3.69 a \pm 0.06$ & $3.59 \mathrm{a} \pm 0.08$ & $3.66 a \pm 0.12$ & $4.99 \mathrm{c} \pm 0.32$ & $5.82 \mathrm{~b} \pm 0.38$ & $6.53 a \pm 0.36$ & $6.29 a b \pm 0.18$ \\
\hline \multirow{4}{*}{ C. iria } & 0 & $2.74 a \pm 0.08$ & 2.61ab0.15 & $2.45 b \pm 0.10$ & $2.43 b \pm 0.04$ & $3.76 \mathrm{a} \pm 0.24$ & $3.69 \mathrm{a} \pm 0.13$ & $3.98 \mathrm{a} \pm 0.06$ & $3.73 a \pm 0.10$ \\
\hline & 20 & $2.93 a \pm 0.02$ & $3.12 \mathrm{a} \pm 0.17$ & $2.91 \mathrm{a} \pm 0.13$ & $2.90 \mathrm{a} \pm 0.03$ & $4.06 \mathrm{~b} \pm 0.34$ & $4.35 \mathrm{ab} \pm 0.25$ & $4.78 \mathrm{a} \pm 0.21$ & $4.45 \mathrm{ab} \pm 0.22$ \\
\hline & 40 & $3.06 \mathrm{~b} \pm 0.06$ & $3.46 a \pm 0.20$ & $3.21 \mathrm{ab} \pm 0.11$ & $3.22 \mathrm{ab} \pm 0.07$ & $4.33 b \pm 0.27$ & $4.92 \mathrm{a} \pm 0.16$ & $5.29 a \pm 0.22$ & $5.01 \mathrm{a} \pm 0.20$ \\
\hline & 60 & $3.44 b \pm 0.10$ & $3.82 \mathrm{a} \pm 0.24$ & $3.72 \mathrm{ab} \pm 0.16$ & $3.74 \mathrm{ab} \pm 0.13$ & $4.75 c \pm 0.26$ & $6.04 b \pm 0.34$ & $6.91 \mathrm{a} \pm 0.15$ & $6.49 \mathrm{ab} \pm 0.21$ \\
\hline
\end{tabular}

Data are expressed as means \pm SD. Values with the same letters in the row for each test weed among the hours after spray are not significantly different at $p>0.05$.
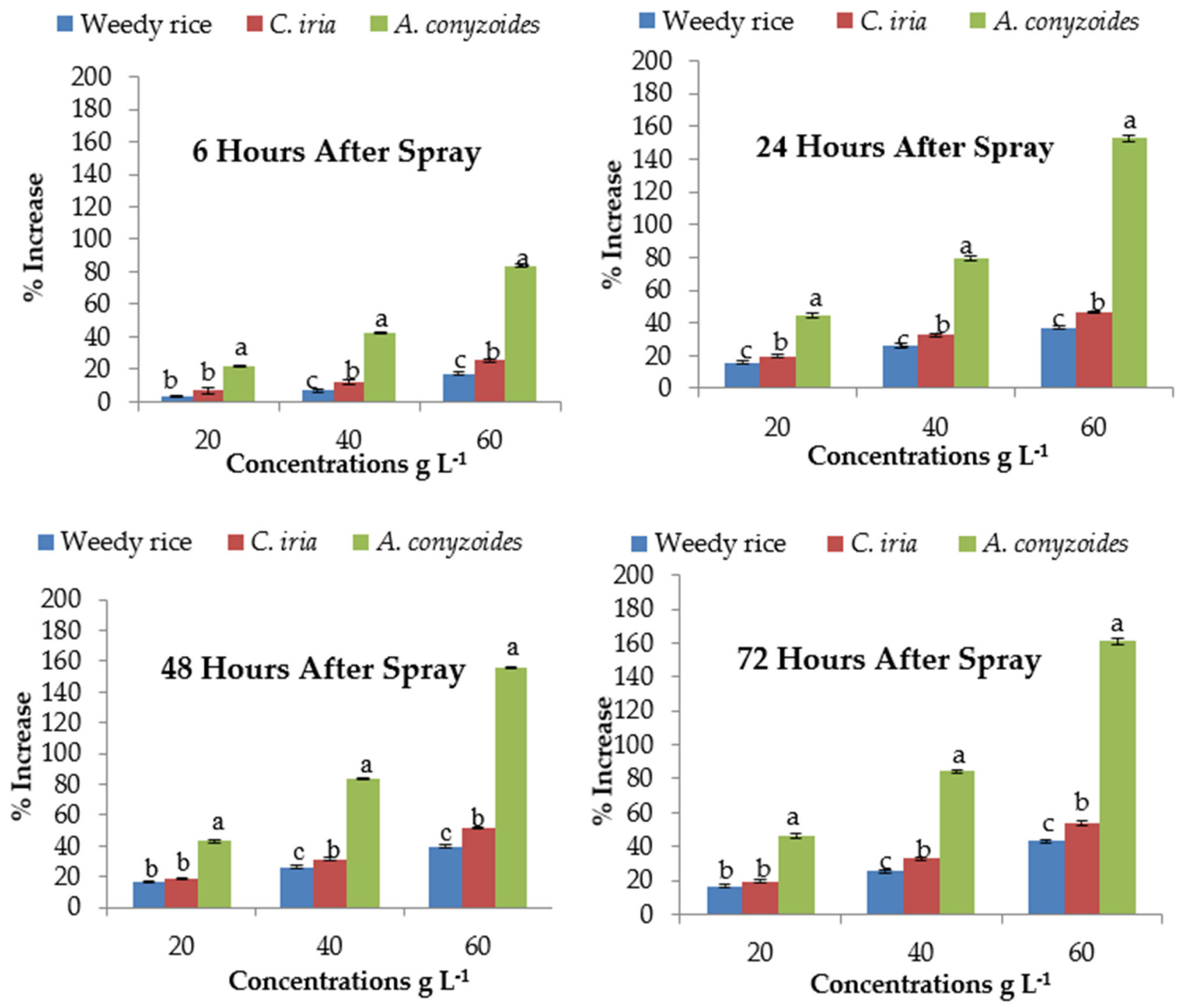

Figure 1. Malondialdehyde (MDA) content (\% increase compared with control) of test weeds treated with P. hyster0ophorus extract concentrations at the different exposure times. Values with the same letter among the test weeds at the same extract concentrations are not significantly different at $p>0.05$ by Tukey's HSD. 


\subsection{Effect of P. hysterophorus Extract on Proline Content of the Test Plant Species}

The proline content was calculated at different exposure times $(6,24,48$ and 72 HAS) and exhibited a significant difference (Table 4). After 6 HAS, the proline content of A. conyzoides was increased by $30.32 \%$ compared to control $\left(0 \mathrm{~g} \mathrm{~L}^{-1}\right)$ at the lowest $\left(20 \mathrm{~g} \mathrm{~L}^{-1}\right)$ concentration, while at the same time, only $4.15 \%$ and $7.92 \%$ increase occurred in weedy rice and C. iria, respectively (Figure 2). The highest $\left(60 \mathrm{~g} \mathrm{~L}^{-1}\right)$ concentration of P. hysterophorus extract exhibited $146.28 \%, 149.73 \%$ and $150.31 \%$ higher proline content than control $\left(0 \mathrm{~g} \mathrm{~L}^{-1}\right)$ at 24,48 and 72 HAS in A. conyzoides, respectively. The increased percentages of proline contents of weedy rice $(55.27 \%)$ and C. iria $(63.66 \%)$ were lower than that of A. conyzoides $(130.32 \%)$ at 24 HAS with the highest $\left(60 \mathrm{~g} \mathrm{~L}^{-1}\right)$ concentration compared to control $\left(0 \mathrm{~g} \mathrm{~L}^{-1}\right)$.
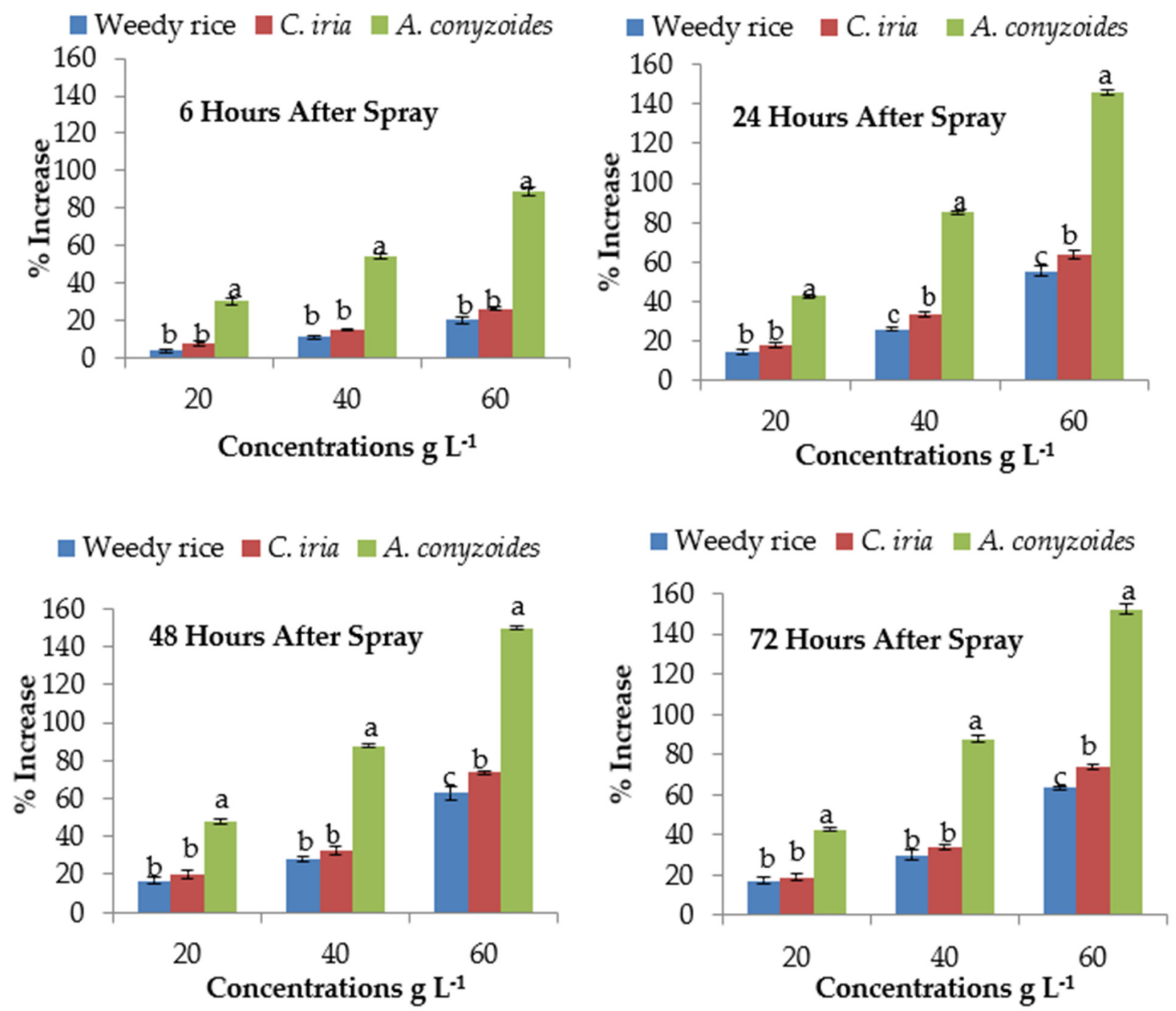

Figure 2. Proline content (\% increase compared with control) of test weeds treated with P. hysterophorus extract concentrations at the different exposure times. Values with the same letter among the test weeds at the same extract concentrations are not significantly different at $p>0.05$ by Tukey's HSD.

2.10. Effect of P. hysterophorus Extract on Superoxide Dismutase of the Test Plant Species

Foliar spray of $P$. hysterophorus extract significantly affected the activity of superoxide dismutase (SOD) enzyme of $A$. conyzoides, weedy rice and C. iria (Table 5). The SOD activity was found the highest with the application of the highest $\left(60 \mathrm{~g} \mathrm{~L}^{-1}\right)$ amount of P. hysterophorus extract. In A. conyzoides, the values were $67.29 \%, 105.87 \%, 108.31 \%$ and 
$116.26 \%$, in weedy ricevalueswere $14.78 \%, 31.21 \%, 34.07 \%$ and $35.06 \%$, and in C. iria they were $19.61 \%, 37.48 \%, 36.72 \%$ and $39.70 \%$ at $6,24,48,72$ HAS, respectively (Figure 3 ). However, the SOD content of $A$. conyzoides was about 68 to $71 \%$ higher than weedy rice and C. iria, respectively, at $24 \mathrm{HAS}$ at the highest $\left(60 \mathrm{~g} \mathrm{~L}^{-1}\right)$ concentration. At the highest concentration $\left(60 \mathrm{~g} \mathrm{~L}^{-1}\right)$, the maximum SOD activity of $A$. conyzoides was 1.67 to 2.16 times higher than the control $\left(0 \mathrm{~g} \mathrm{~L}^{-1}\right)$ of different exposure times $(6,24,48$ and 72$)$. At 72 HAS, the SOD activity of weedy rice and C. iria was increased by 6.44 to $35.06 \%$ and 9.38 to $39.70 \%$ at the lowest $\left(20 \mathrm{~g} \mathrm{~L}^{-1}\right)$ and highest $\left(60 \mathrm{~g} \mathrm{~L}^{-1}\right)$ concentration compared to control $\left(0 \mathrm{~g} \mathrm{~L}^{-1}\right)$, respectively.
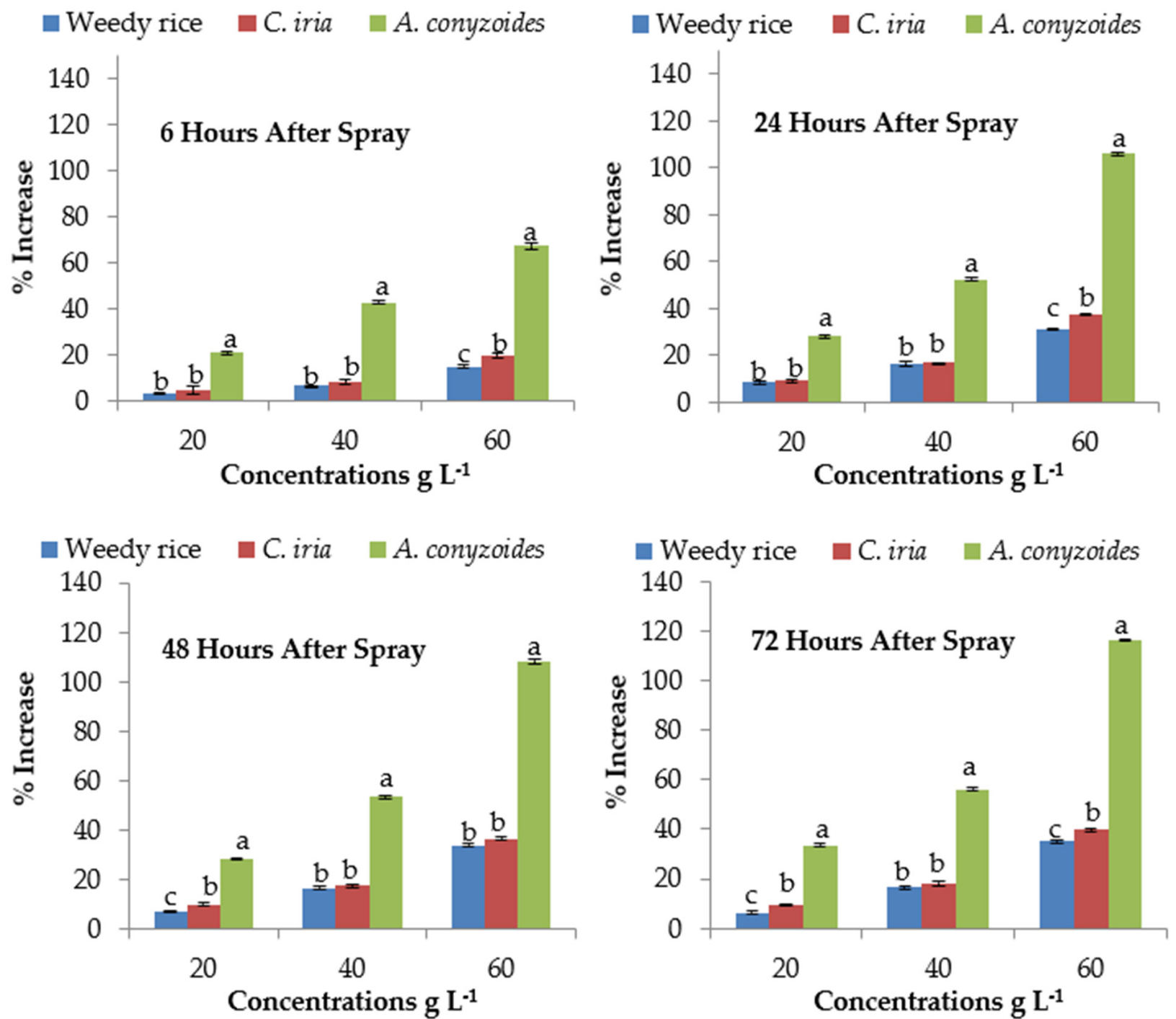

Figure 3. Activities of superoxide dismutase (SOD) (\% increase compared with control) of test weeds treated with P. hysterophorus extract concentrations at the different exposure times. Values with the same letter among the test weeds at the same extract concentrations are not significantly different at $p>0.05$ by Tukey's HSD. 
Table 5. Response of superoxide dismutase, catalase and peroxidase activity of A. conyzoides, weedy rice and C. iria to a foliar spray of $P$. hysterophorus extract.

\begin{tabular}{|c|c|c|c|c|c|c|c|c|c|c|c|c|c|}
\hline \multirow{3}{*}{ Test Plants } & \multirow{3}{*}{$\begin{array}{l}\text { Dose } \\
\left(\mathrm{g} \mathrm{L}^{-1}\right)\end{array}$} & \multicolumn{4}{|c|}{ Superoxide Dismutase (Unit g ${ }^{-1} \mathrm{FW}$ ) } & \multicolumn{4}{|c|}{ Catalase $\left(\mu \mathrm{mol} \mathrm{g}{ }^{-1}\right.$ FW) } & \multicolumn{4}{|c|}{ Peroxidase $\left(\mu \mathrm{mol} \mathrm{g}^{-1} \mathrm{FW}\right)$} \\
\hline & & \multirow{2}{*}{\multicolumn{3}{|c|}{ Hours after Spray }} & \multirow[b]{2}{*}{72} & \multicolumn{4}{|c|}{ Hours after Spray } & \multicolumn{4}{|c|}{ Hours after Spray } \\
\hline & & & 24 & 48 & & 6 & 24 & 48 & 72 & 6 & 24 & 48 & 72 \\
\hline \multirow{4}{*}{$\begin{array}{c}\text { A. } \\
\text { conyzoides }\end{array}$} & 0 & $3.40 \mathrm{a} \pm 0.07$ & $3.35 a \pm 0.10$ & $3.31 \mathrm{a} \pm 0.04$ & $3.29 a \pm 0.29$ & $5.34 \mathrm{a} \pm 0.20$ & $5.15 a \pm 0.08$ & $5.09 \mathrm{a} \pm 0.11$ & $5.04 a \pm 0.18$ & $11.00 \mathrm{c} \pm 1.93$ & \multirow{2}{*}{$\begin{array}{c}12.77 \mathrm{a} \pm 1.18 \\
16.66 \mathrm{ab} \pm \\
1.48\end{array}$} & \multirow{2}{*}{$\begin{array}{c}10.15 \mathrm{a} \pm 0.92 \\
13.79 \mathrm{bc} \pm \\
1.11\end{array}$} & $12.72 \mathrm{a} \pm 0.63$ \\
\hline & 20 & $4.11 \mathrm{a} \pm 0.12$ & $4.28 \mathrm{a} \pm 0.15$ & $4.25 a \pm 0.08$ & $4.39 a \pm 0.38$ & $6.00 \mathrm{a} \pm 0.19$ & $6.18 \mathrm{a} \pm 0.03$ & $6.26 \mathrm{a} \pm 0.13$ & $6.15 a \pm 0.20$ & $12.38 \mathrm{c} \pm 2.20$ & & & $16.94 \mathrm{a} \pm 0.72$ \\
\hline & 40 & $4.85 a \pm 0.11$ & $5.10 \mathrm{a} \pm 0.12$ & $5.07 \mathrm{a} \pm 0.01$ & $5.13 a \pm 0.46$ & $6.48 \mathrm{~b} \pm 0.28$ & $7.27 \mathrm{a} \pm 0.19$ & $7.35 \mathrm{a} \pm 0.15$ & $7.41 \mathrm{a} \pm 0.29$ & $14.15 b \pm 2.61$ & $21.82 \mathrm{a} \pm 1.93$ & $17.40 \mathrm{~b} \pm 1.66$ & $21.96 \mathrm{a} \pm 0.87$ \\
\hline & 60 & $5.69 \mathrm{~b} \pm 0.10$ & $6.89 \mathrm{a} \pm 0.27$ & $6.87 \mathrm{a} \pm 0.06$ & $7.11 \mathrm{a} \pm 0.63$ & $7.12 b \pm 0.36$ & $8.18 \mathrm{a} \pm 0.06$ & $8.31 \mathrm{a} \pm 0.17$ & $8.28 \mathrm{a} \pm 0.25$ & $11.23 c \pm 3.08$ & $27.63 a \pm 2.37$ & $22.47 \mathrm{~b} \pm 1.93$ & $28.22 \mathrm{a} \pm 1.45$ \\
\hline \multirow{4}{*}{ Weedy rice } & 0 & $4.01 \mathrm{a} \pm 0.06$ & $3.98 \mathrm{a} \pm 0.04$ & $3.79 \mathrm{a} \pm 0.15$ & $3.83 a \pm 0.17$ & $5.88 \mathrm{a} \pm 0.43$ & $5.52 \mathrm{a} \pm 0.25$ & $5.86 \mathrm{a} \pm 0.27$ & $\begin{array}{c}5 . .44 \mathrm{a} \pm \\
0.37\end{array}$ & $19.74 a \pm 1.46$ & $21.15 \mathrm{a} \pm 1.08$ & $21.71 \mathrm{a} \pm 1.93$ & $21.79 \mathrm{a} \pm 1.60$ \\
\hline & 20 & $4.14 \mathrm{a} \pm 0.07$ & $4.31 \mathrm{a} \pm 0.03$ & $4.05 a \pm 0.15$ & $4.07 a \pm 0.22$ & $6.02 \mathrm{a} \pm 0.38$ & $5.80 \mathrm{a} \pm 0.23$ & $6.23 a \pm 0.31$ & $5.81 \mathrm{a} \pm 0.36$ & $20.22 b \pm 1.57$ & $23.94 \mathrm{a} \pm 1.26$ & $25.07 \mathrm{a} \pm 1.91$ & $25.15 a \pm 1.65$ \\
\hline & 40 & $4.27 \mathrm{~b} \pm 0.06$ & $4.63 a \pm 0.07$ & $\begin{array}{c}4.42 \mathrm{ab} \pm \\
0.12\end{array}$ & $\begin{array}{c}4.45 \mathrm{ab} \pm \\
0.17\end{array}$ & $6.16 \mathrm{a} \pm 0.42$ & $6.26 a \pm 0.28$ & $6.70 \mathrm{a} \pm 0.34$ & $6.20 \mathrm{a} \pm 0.34$ & $20.86 \mathrm{~b} \pm 1.45$ & $26.70 \mathrm{a} \pm 1.34$ & $28.45 a \pm 2.32$ & $29.63 a \pm 1.82$ \\
\hline & 60 & $4.60 \mathrm{~b} \pm 0.12$ & $5.22 \mathrm{a} \pm 0.06$ & $5.08 a \pm 0.18$ & $5.17 a \pm 0.18$ & $6.31 \mathrm{a} \pm 0.40$ & $6.77 a \pm 0.33$ & $7.08 \mathrm{a} \pm 0.30$ & $6.69 a \pm 0.48$ & $21.99 b \pm 1.46$ & $32.06 a \pm 1.47$ & $35.05 a \pm 3.12$ & $36.09 a \pm 2.72$ \\
\hline \multirow[b]{3}{*}{ C. iria } & 0 & $3.99 a \pm 0.06$ & $3.67 \mathrm{c} \pm 0.06$ & $\begin{array}{c}3.85 \mathrm{ab} \pm \\
0.13\end{array}$ & $\begin{array}{c}3.73 b c \pm \\
0.03\end{array}$ & $6.02 \mathrm{a} \pm 0.08$ & $\begin{array}{c}5.64 \mathrm{ab} \pm \\
0.34\end{array}$ & $\begin{array}{c}5.47 \mathrm{ab} \pm \\
0.28\end{array}$ & $5.46 \mathrm{~b} \pm 0.29$ & $17.48 \mathrm{~b} \pm 1.46$ & $21.43 a \pm 0.92$ & $19.96 a \pm 0.54$ & $21.40 \mathrm{a} \pm 0.92$ \\
\hline & 20 & $\begin{array}{c}4.17 a b \pm \\
0.11\end{array}$ & $4.01 \mathrm{~b} \pm 0.05$ & $4.23 a \pm 0.11$ & $\begin{array}{c}4.08 \mathrm{ab} \pm \\
0.01\end{array}$ & $6.21 \mathrm{a} \pm 0.12$ & $6.11 \mathrm{a} \pm 0.35$ & $5.86 \mathrm{a} \pm 0.30$ & $6.01 \mathrm{a} \pm 0.32$ & $18.16 \mathrm{~b} \pm 1.40$ & $24.87 \mathrm{a} \pm 0.89$ & $24.56 a \pm 0.71$ & $26.31 \mathrm{a} \pm 1.45$ \\
\hline & 40 & $4.31 \mathrm{~b} \pm 0.05$ & $4.29 b \pm 0.06$ & $4.53 a \pm 0.14$ & $\begin{array}{c}4.41 \mathrm{ab} \pm \\
0.05\end{array}$ & $6.37 \mathrm{a} \pm 0.05$ & $6.49 \mathrm{a} \pm 0.34$ & $6.48 a \pm 0.28$ & $6.60 \mathrm{a} \pm 0.31$ & $18.61 \mathrm{c} \pm 1.46$ & $\begin{array}{c}28.25 \mathrm{ab} \pm \\
0.93\end{array}$ & $27.21 b \pm 0.54$ & $29.80 \mathrm{a} \pm 1.21$ \\
\hline
\end{tabular}

Data are expressed as means \pm SD. Values with the same letters in the row among the hours after spray for each test weed are not significantly different at $p>0.05$. 


\subsection{Effect of P. hysterophorus Extract on Catalase Activity of the Test Plant Species}

Catalase (CAT) activity differed significantly with increasing P. hysterophorus extract concentration at different exposure times except for weedy rice (Table 5). At the lowest $\left(20 \mathrm{~g} \mathrm{~L}^{-1}\right)$ concentration, CAT activity of $A$. conyzoides showed 12.47, 20.04, 22.99 and $22.08 \%$ increases after $6,24,48$ and 72 HAS respectively compared to control $\left(0 \mathrm{~g} \mathrm{~L}^{-1}\right)$. These activities increased to $1.33,1.59,1.63$ and 1.64 times than that of the control $\left(0 \mathrm{~g} \mathrm{~L}^{-1}\right)$ at the highest $\left(60 \mathrm{~g} \mathrm{~L}^{-1}\right)$ P. hysterophorus concentration after 6, 24, 48 and $72 \mathrm{~h}$, respectively in A. conyzoides. The CAT activity of weedy rice was increased only 2.46 to $7.40 \%$ at 6 HAS, while $6.80 \%$ and $23.06 \%$ increases occurred at 72 HAS in the lowest $\left(20 \mathrm{~g} \mathrm{~L}^{-1}\right)$ and highest $\left(60 \mathrm{~g} \mathrm{~L}^{-1}\right)$ concentration, respectively, compared to control $\left(0 \mathrm{~g} \mathrm{~L}^{-1}\right)$ (Figure 4$)$. The highest CAT activity was observed at 72 HAS for C. iria with the highest amount of P. hysterophorus, and the increasing percentage was 29.39. No significant differences were recorded in C. iria and weedy rice among different exposure times of $P$. hysterophorus extract concentrations.
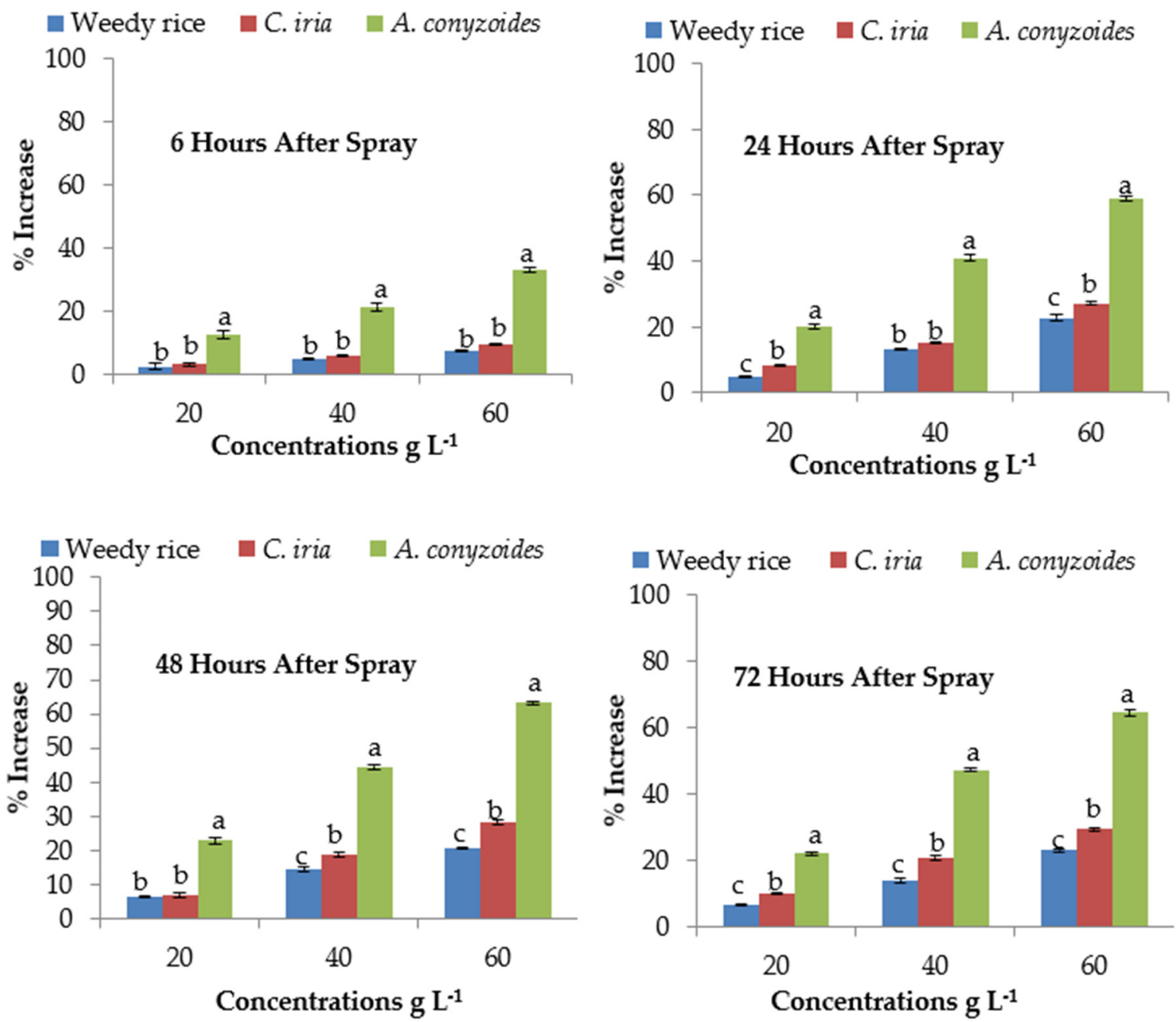

Figure 4. Activities of catalase (CAT) (\% increase compared with control) of test weeds treated with P. hysterophorus extract concentrations at the different exposure times. Values with the same letter among the tested weed at the same extract concentrations are not significantly different at $p>0.05$ by Tukey's HSD.

\subsection{Effect of P. hysterophorus Extract on Peroxidase Activity of the Test Plant Species}

The interaction of exposure time and P. hysterophorus extract significantly influenced the peroxidase (POD) activity of $A$. conyzoides, weedy rice and $C$. iria (Table 5). The POD activity of all test plant species was significantly different at different exposure times. Longer exposure times of spray (i.e., 24,48 and $72 \mathrm{~h}$ ) induced a significant increase in 
the POD activity (Figure 5). The POD activity increased significantly with the increase in concentration where the greatest activity $(116.43 \%)$ was recorded in $60 \mathrm{~g} \mathrm{~L}^{-1}$, while 40 and $20 \mathrm{~g} \mathrm{~L}^{-1}$ concentrations recorded $70.91 \%$ and $30.50 \%$ increase at 24 HAS in A. conyzoides compared to control. On the other hand, at $24 \mathrm{HAS}$, POD activities of weedy rice and C. iria were increased by $51.63 \%$ and $66.71 \%$, respectively, at the highest $\left(60 \mathrm{~g} \mathrm{~L}^{-1}\right)$ concentration of $P$. hysterophorus extract compared to control $\left(0 \mathrm{~g} \mathrm{~L}^{-1}\right)$. The increased percentage of POD activity was 33.30 to $121.94 \%, 15.39$ to $65.59 \%$ and 22.88 to $70.39 \%$ in A. conyzoides, weedy rice and C. iria, respectively, at the highest exposure time (72 HAS) compared to control.
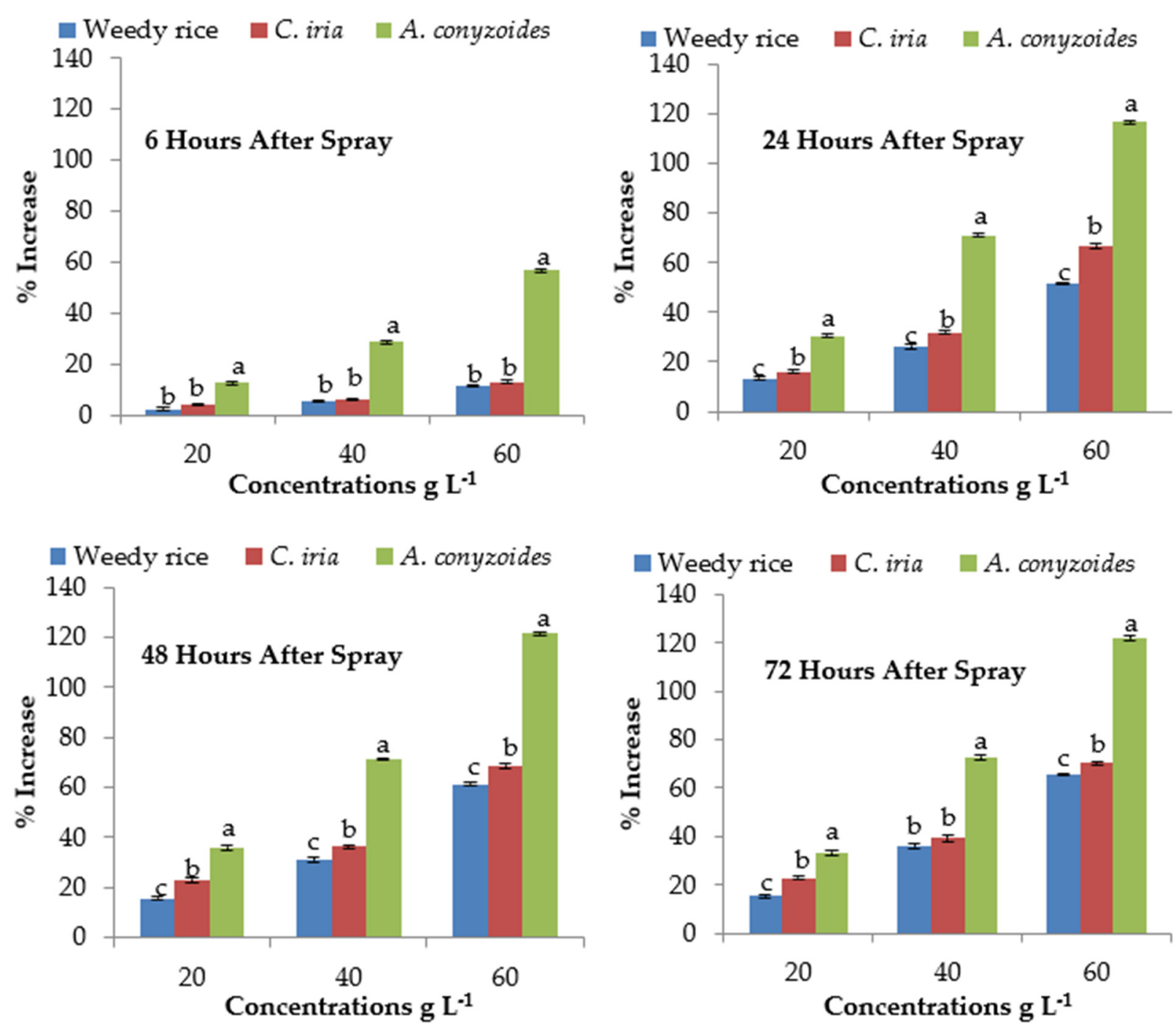

Figure 5. Activities of peroxidase (POD) (\% increase compared with control) of test weeds treated with P. hysterophorus extract concentrations at the different exposure times. Values with the same letter among the tested weed at the same extract concentrations are not significantly different at $p>0.05$ by Tukey's HSD.

\section{Discussion}

Parthenium hysterophorus has been spreading like wildfire in different countries and creates harm, including threats to biodiversity, allergies, mutagenesis and dermatitis in livestock and humans. In this study, we observed the effect of P. hysterophorus extract on physiological and biochemical changes in A. conyzoides, weedy rice and C. iria at different times of exposure.

Changes in chlorophyll levels in leaves are an important indicator of plant resistance and environmental quality [20]. In this study, we found a significant decrease in the 
treatment groups compared to the control groups. This decrease in the chlorophyll content is due to the allelopathic effect of P. hysterophorus. The decreased chlorophyll and carotenoid contents depend on the concentration of $P$. hysterophorus extract, and these tallies with the finding of Ding et al. [21], who observed that a high concentration of allelochemicals inhibits photosynthesis and plant growth by reducing chlorophyll content. Yilmaz et al. [22] and Erdal [23] have explained that decreased chlorophyll content during stress conditions is associated with an increase in the chlorophyllase enzyme. Carotenoids served as an antioxidant against free radicals and photochemical damage [24]. Chlorophyll $b$ and carotenoids were decreased by $81.40 \%$ and $77.8 \%$ when exposed to Portulaca oleracea root extract, and these could be attributed to a decrease in chlorophyll biosynthesis or degradation of existing chlorophyll [25].

Foliar spray of $P$. hysterophorus extract reduced photosynthesis rate, stomatal conductance and transpiration of $A$. conyzoides, weedy rice and $C$. iria. Reduction in leaf photosynthesis was attributed to a decrease in photosynthetic metabolites, carboxylation efficiency, impairment of chloroplast activity, increase in enzyme activities [26], and production of ROS caused impediment of photosynthetic mechanism [27]. Stomatal control is an important property through which the plants limit the loss of water, affecting gas changes. This characteristic can be influenced by several factors, including stress [28], and it can be an indication of lower photosynthetic efficiency. The efficiency of using water and carboxylation was also reduced in the plants subjected to the application of P. hysterophorus extract.

The reduction in the transpiration rate is certainly associated with stomatal conductance. This study reveals that $P$. hysterophorus extract played a notable role in decreasing the transpiration rate for test plants at different exposure times. The concentration of phenolic acids resulted in a decline in overall water utilization and transpiration of cucumber seedlings in a linear manner [29]. The solution of cinnamic acid and benzoic acids decreased stomatal conductance and transpiration of cucumber seedlings [30]. The results obtained for the variables related to photosynthesis corroborate the ones obtained for phytotoxicity, as phytotoxicity caused by the increased amount of $P$. hysterophorus extract and exposure time, there was a reduction in the photosynthesis, stomatal conductance and transpiration rate.

In the present study, P. hysterophorus exposures increase the activities of SOD, POD and CAT. SOD is the first step in the removal of ROS. Specifically, it converts $\mathrm{O}_{2}{ }^{-}$to $\mathrm{H}_{2} \mathrm{O}$ and oxygen. Therefore, the increases in the activity of SOD in response to P. hysterophorus suggest an increased production of $\mathrm{O}_{2}{ }^{-}$. Similarly, the increased activities of POD and CAT indicate potential protection against oxidation by this antioxidant enzyme [31]. In this study, SOD, POD and CAT activities in the leaves of A. conyzoides were significantly increased than weedy rice and C. iria at higher concentrations. The activities of one or more of these enzymes generally increase when plants are exposed to stressful conditions [32]. Ding et al. [21] reported the toxicity of allelochemicals from garlic root exudates on the activity of protective enzymes might be due to their intra-structure disruption.

In this study, the enzymatic activities of $A$. conyzoides at 72 HAS were stimulated significantly at higher concentrations to $64.41 \%$ and $120.18 \%$, respectively. The high overwhelming increase in POD indicated an abundant high level of $\mathrm{H}_{2} \mathrm{O}_{2}$, which is a byproduct of SOD metabolism. Generally, the activity of the enzyme increases with increasing $P$. hysterophorus extract concentration. It appears that the enzymes (SOD, CAT and POD) were not adequate to scavenge ROS and prevent membrane oxidation in the organelles, especially mitochondria and chloroplast, at the initial period of $P$. hysterophorus extract application. In addition, chlorotic symptoms revealed signal molecules activation and secretion of defense molecules against allelochemical stress. The recovery action observed in A. conyzoides signifies the rapid production of ROS.

An increased malondialdehyde (MDA) level in the plant tissue represents an important indicator of membrane lipid peroxidation. In our study, the MDA content increase in $P$. hysterophorus sprayed plants compared to control (without extract) plants, and this 
increase was more apparent in the highest concentration. P. hysterophorus may induce the formation of free radicals by inducing the degradation of carotenoids and the indirect degradation of chlorophyll. Furthermore, increased free radicals level can increase membrane lipid peroxidation and thus MDA content. Saidi et al. [33] reported that the bean plants exposed to $20 \mu \mathrm{M}$ cadmium increased MDA levels compared to control plants. Choudhury and Panda [34] stated that an increase in MDA content is an indicator of oxidative stress. These findings are consistent with our result. The consistently high level of MDA suggests that the antioxidant enzymes induced by glufosinate may not be able to completely eliminate ROS within a short period of time.

Proline is the amino acid that is associated with different stresses in the plant. Under abiotic stress, accumulation of proline in the plant may be an adaptive and metabolic measure of stress, an inhibitor of lipid peroxidation and defense against toxicity [35]. Plants develop mechanisms for the accumulation of compatible solutes, such as betaine, sugar, polyol and proline to counteract various abiotic stress, and among them, proline is the most important solutes that curtail the impact of osmotic adjustment [36]. The greatest content of proline in 72 HAS was observed in the treatment with P. hysterophorus. Weedy rice and C. iria had the lowest proline content compared to A. conyzoides. This was expected considering stress and leaf damage that was visually observed during experimentation. In a similar relation, foliar application of Medicago sativa leaf extracts significantly raised the proline content of three wheat varieties [37]. Proline content of tomato, wheat and cucumber treated with aqueous extract of Calotropis procera were stimulated with an increase in concentration [38].

\section{Materials and Methods}

\subsection{Experimental Site}

The experiment was conducted in a glasshouse in Farm 15 at the Faculty of Agriculture Universiti Putra Malaysia $\left(3^{\circ} 02^{\prime} \mathrm{N}, 101^{\circ} 42^{\prime} \mathrm{E}, 31 \mathrm{~m}\right.$ elevation). The climate was hot (on average $36-38^{\circ} \mathrm{C}$ ) and humid with abundant rainfall during the experimental period.

\subsection{Test Plants}

Three weed species, namely A. conyzoides (voucher specimen\#UPMWS001), C. iria (voucher specimen\#UPMWS019) and weedy rice (voucher specimen\#UPMWS025), were used as test plants. The seeds of weedy rice were collected from the rice field of Sekinchan, Kuala Selangor, Selangor, Malaysia, and other weed seeds (A. conzyoides and C. iria) were collected from farm 15, Universiti Putra Malaysia. The voucher specimens are deposited in the Weed Science Laboratory, Department of Crop Science, Faculty of Agriculture, Universiti Putra Malaysia.

\subsection{Preparation of P. hysterophorus Extract for Foliar Spray}

The whole plant of $P$. hysterophorus at the maximum vegetative stage was collected. The collected weed was washed carefully with running tap water to remove the dust particles, then air-dried in open trays under shade at room temperature for 3 weeks. Then chopped and powdered in a Willey mill. In a conical flask, $100 \mathrm{~g}$ powder of $P$. hysterophorus was soaked with $1000 \mathrm{~mL}$ methanol: distilled water (80:20,v/v) and paraffin were used for wrapping the flask. The flask was shaken in an orbital shaker at $150 \mathrm{rpm}$ agitation speed for $48 \mathrm{~h}$ at room temperature $\left(24-26^{\circ} \mathrm{C}\right)$. The solution was filtered using four layers of cheesecloth, then centrifuged for $1 \mathrm{~h}$ at $3000 \mathrm{rpm}$, then re-filtered using $0.2 \mu \mathrm{m}, 15 \mathrm{~mm}$ syringe filters (Phenex, Nonsterile, Luer/Slip, LT Resources, Malaysia). The collected supernatant was evaporated by a rotary evaporator at $40{ }^{\circ} \mathrm{C}$. The dried residue was weighed and converted to \% as under:

$$
\text { Extraction percentage }=[\text { Extract weight }(\mathrm{g}) / \text { powder weight }(\mathrm{g})] \times 100 \%
$$

Each stock extract was diluted with sterile distilled water to get 20,40,60 $\mathrm{g} \mathrm{L}^{-1}$ concentrations of extract for bioassay. All extracts were stored in the dark at $4{ }^{\circ} \mathrm{C}$ in 
the refrigerator until used. The methanol extracts were prepared as per the method of Motmainna et al. [39].

\subsection{Experimental Layout}

Pre-germinated seeds of $A$. conyzoides, weedy rice and $C$. iria were seeded in each pot (15 cm diameter) and then covered with soil at a depth of $1 \mathrm{~cm}$, and finally, the soil was moistened with tap water. After germination, equal-sized healthy five seedlings were maintained in each pot. The pots were arranged in a randomized complete block design with four replications. The methanol extract of $P$. hysterophorus was sprayed with 20,40 or $60 \mathrm{~g} \mathrm{~L}^{-1}$ concentration on tested plants (4-6 for broadleaf and 2-3 leaf stage for grasses and sedges species) with the help of a hand-operated atomizer at the rate of $100 \mathrm{~mL} \mathrm{~m}^{-2}$ [40]. Plants in the control treatment were sprayed with $200 \mathrm{~mL}$ water without extract at two-day intervals or when needed.

\subsection{Data Collections}

The physiological and biochemical changes due to the application of P. hysterophorus extract on the test weed species were observed to elucidate the possible mechanism of its allelopathy data on photosynthesis rate, transpiration and stomatal conductance, chlorophyll fluorescence, chlorophyll pigments, proline and antioxidant enzymes. Mature leaves of each test weed species were plucked at 6, 24, 48 and $72 \mathrm{~h}$ after spray and kept inside aluminum foil and brought to the laboratory from glasshouse through icebox. The leaf samples were harvested between 8 to 10 am and rapidly frozen with liquid nitrogen and then stored at $-80^{\circ} \mathrm{C}$ for further biochemical analysis.

\subsubsection{Photosynthesis Rate, Transpiration and Stomatal Conductance}

The rate of photosynthesis, transpiration and stomatal conductance were measured from randomly selected four leaves from each test weed species using LICOR (LI-6400XT) portable photosynthesis system (LI-COR-Inc Lincoln, Lincoln, NE, USA) between $9.00 \mathrm{am}$ to $11.00 \mathrm{am}$ under bright daylight. The measurements were taken on the abaxial surface at a $\mathrm{CO}_{2}$ flow rate of $400 \mu \mathrm{mol} \mathrm{m}{ }^{-2} \mathrm{~s}^{-1}$, and the saturating photosynthetic photon flux density (PPFD) was $1000 \mathrm{mmol} \mathrm{m}^{-2} \mathrm{~s}^{-1}$ [36].

\subsubsection{Chlorophyll Pigments}

The total chlorophyll and carotenoids contents were determined following the procedure of Lichenthaler and Bushman [41] and Amin [42]. The samples of the fresh leaf $(0.1 \mathrm{~g})$ were homogenized into a glass bottle having an amount of $10 \mathrm{~mL}$ of $80 \%$ acetone. The glass bottles were covered by aluminum foil and kept in the dark place for three to four days at room temperature. After finishing the incubation, the test tubes were vortexed and needed to wait until the sediments have found at the bottom. The spectrophotometric reading (UV-3101 P, Labomed Inc., Angeles, CA, USA) of the absorbance of the solution was measured at $663.2 \mathrm{~nm}, 646.8 \mathrm{~nm}$ and $470 \mathrm{~nm}$, and as a blank, $80 \%$ acetone was used. Chlorophyll- $a$, chlorophyll- $b$, total chlorophyll and carotenoids contents were expressed as $\mathrm{mg} \mathrm{g}^{-1}$ of fresh weight (FW) using the following relationships:

$$
\begin{gathered}
\text { Chlorophyll- } a\left(\mu \mathrm{g} \mathrm{mL}^{-1}\right)=(12.25 \times \mathrm{A} 663.2-2.79 \times \mathrm{A} 646.8) \\
\text { Chlorophyll- } b\left(\mu \mathrm{g} \mathrm{mL}^{-1}\right)=(21.50 \times \mathrm{A} 646.8-5.1 \times \mathrm{A} 663.2) \\
\text { Total chlorophyll }\left(\mu \mathrm{g} \mathrm{mL}{ }^{-1}\right)=(7.15 \times \mathrm{A} 663.2+18.71 \times \mathrm{A} 646.8) \\
\text { Carotenoids }\left(\mu \mathrm{g} \mathrm{mL}^{-1}\right)=\frac{(1000 \times \mathrm{A} 470-1.8 \times \mathrm{chl} a-85.02 \times \mathrm{chl} b)}{198}
\end{gathered}
$$

\subsubsection{Malondialdehyde (MDA) Content}

The malondialdehyde (MDA) content was determined by the following protocol as previously described by Robert [43]. An amount of $0.2 \mathrm{~g}$ of grounded leaf tissue was 
homogenized in $2 \mathrm{~mL}$ ultrapure distilled water. Then the samples were centrifuged (Sigma $3 \mathrm{~K} 30$ ) at 10,000 rpm for $15 \mathrm{~min}$. One milliliter of this solution and $2 \mathrm{~mL}$ of thiobarbituric acid (TBA)/trichloroacetic acid (TCA) (Merck, German) solution (0.5\% TBA in 20\% TCA) were boiled in a water bath at $90^{\circ} \mathrm{C}$ for $30 \mathrm{~min}$. After boiling, the test tubes containing the solution were cooled in an ice bath. The final mixture was centrifuged again at 10,000 rpm for $15 \mathrm{~min}$. The spectrophotometric absorbance (UV-3101PC, Shimadzu) of the supernatants was recorded at 450, 532 and $600 \mathrm{~nm}$. The MDA concentration was measured using the following relationship:

$$
\operatorname{MDA}(\mu \mathrm{M})=\{6.45 \times(\mathrm{D} 532-\mathrm{D} 600)-0.56 \times \mathrm{D} 450\}
$$

where D450, D532 and D600 were the absorbances at 450, 532 and $600 \mathrm{~nm}$, respectively. The MDA content was finally expressed as $\mu \mathrm{mol} \mathrm{g}^{-1} \mathrm{FW}$.

\subsubsection{Proline Content}

Proline measurement protocol was done according to methods described by Bates et al. [44] with slight modifications. An amount of $0.1 \mathrm{~g}$ of fresh leaves was homogenized with the presence of $2 \mathrm{~mL}$ of $5 \%(w / v)$ sulfosalicylic acid. Sample containing test tubes were centrifuged at 10,000 rpm for $10 \mathrm{~min}$. One milliliter of the supernatant was added in one milliliter of acid ninhydrin $(1.25 \mathrm{~g}$ of ninhydrin; $30 \mathrm{~mL}$ of glacial acetic acid; $20 \mathrm{~mL}$ of phosphoric acid $6 \mathrm{M}$ ) and one milliliter of glacial acetic acid. Then the solution containing tubes was incubated at $95^{\circ} \mathrm{C}$ for one hour in a water bath and cooled for $10 \mathrm{~min}$ in an ice bath. Two milliliters of toluene was added in each tube with samples then shaken using a vortex. The proline concentration was recorded by taking the absorbance reading at $520 \mathrm{~nm}$ using the microplate reader (Bio Tek 800 TS). The content of proline was calculated by plotting the value in a standard curve considering L-proline as standard (Sigma-Aldrich, St. Louis, MO, USA), then proline content was calculated following the equation stated below:

$$
\text { Proline }\left(\mu \mathrm{molg}^{-1} \mathrm{FW}\right)=\frac{\left.\frac{\text { Proline }\left(\mu \mathrm{g} \mathrm{mL}^{-1}\right) \times \text { Tolune }^{-}(\mathrm{mL})}{115.5(\mu \mathrm{g} \mathrm{\mu mole}}-1\right)}{\text { Freshweightofsample }(\mathrm{g})}
$$

where the molecular weight of proline is $115.5\left(\mu \mathrm{g} \mu \mathrm{mole}^{-1}\right)$.

\subsubsection{Enzymes Extraction}

The enzyme extraction was performed based on the protocol described by Gupta et al. [45] to determine the activity of the antioxidant enzymes, superoxide dismutase (SOD), catalase (CAT), and peroxidase (POD). Fresh leaf sample was ground in a porcelain mortar with liquid nitrogen. Then $0.1 \mathrm{~g}$ ground leaf sample was taken into $2 \mathrm{~mL}$ Eppendorf tube, and $1.5 \mathrm{~mL}$ potassium phosphate buffer was added. The mixture was centrifuged at 10,000 rpm for $20 \mathrm{~min}$.

\section{Determination of SOD}

The reaction mixture was prepared by adopting the method described by Gupta et al. [45]. The reaction mixture contained $0.05 \mathrm{~mL}$ of the enzyme extract, $1.5 \mathrm{~mL}$ of $100 \mathrm{mM}$ potassium phosphate buffer, $0.1 \mathrm{~mL}$ of $3 \mathrm{mM}$ EDTA, $0.1 \mathrm{~mL}$ of $200 \mathrm{mM}$ methionine, $0.01 \mathrm{~mL} 2.25 \mathrm{mM}$ NBT (n-nitro blue tetrazolium) and $1 \mathrm{~mL}$ of ultra-pure distilled water. Finally, $60 \mu \mathrm{M}$ of riboflavin was added into each reaction mixture in the dark. Then the tubes were incubated in a 15 watts fluorescent lamp for $10 \mathrm{~min}$. The control mixture without enzyme extract was kept under the light. The blank consisted of reaction mixtures with no enzyme extract and without keeping under the light to stop the reaction immediately after incubation. Aluminum foil was used to cover the tubes; SOD was measured using a spectrophotometer (UV-3101PC) at $560 \mathrm{~nm}$ through recording the change in absorbance due to the reaction of the superoxide nitro blue tetrazolium complex with enzyme extracts. Each unit of enzyme 
activity was calculated by the amount of enzyme that inhibits NBT reduction to $50 \%$ using the formula described as follows:

The activity of SOD can be expressed as unit $\mathrm{mg}^{-1} \mathrm{FW}$ :

$$
\begin{gathered}
\text { SOD }(\% \text { inhibition })=\frac{(\text { A560 control }- \text { A560 sample }) \times 100}{\text { A560 control }} \\
\text { SOD }\left(\text { unit } \mathrm{mL}^{-1}\right)=\frac{\% \text { inhibition } \times \text { total volume }}{50 \times \text { enzyme volume }} \\
\text { SOD }\left(\text { unit } \mathrm{mg}^{-1} \mathrm{FW}\right)=\frac{\text { unit } \mathrm{mL}^{-1}}{\text { enzyme }\left(\mathrm{mg} \mathrm{mL}^{-1}\right)}
\end{gathered}
$$

where the absorbance of control and sample was recorded by $560 \mathrm{~nm}$ at $1 \mathrm{~min}, 50 \%$ inhibition is equal to 1 unit of SOD production.

\section{Determination of CAT}

The CAT activity was studied considering the methodology stated by Aebi [46]. A $3.0 \mathrm{~mL}$ of reaction mixture contained $1.5 \mathrm{~mL}$ of $100 \mathrm{mM}$ potassium phosphate buffer, $0.5 \mathrm{~mL}$ of $75 \mathrm{mM}$ hydrogen peroxide $\left(\mathrm{H}_{2} \mathrm{O}_{2}\right), 0.05 \mathrm{~mL}$ of enzyme extract and $0.95 \mathrm{~mL}$ of ultrapure distilled water. The mixture with no enzyme extract was considered blank. To reach the temperature equilibration, the blank solution was put in a spectrophotometer for 4 to $5 \mathrm{~min}$. The absorbance reading in a spectrophotometer (UV-3101PC, Shimadzu, Japan) at a wavelength of $240 \mathrm{~nm}$ was done for $2 \mathrm{~min}$. Each unit of catalase enzyme activity was considered as the amount that decomposes $1 \mu \mathrm{M} \mathrm{H}_{2} \mathrm{O}_{2}$. The unit of catalase action was expressed as per $\mathrm{g}$ of fresh tissue $\left(\mu \mathrm{Mmin}^{-1} \mathrm{~g}^{-1} \mathrm{FW}\right)$.

$$
\begin{aligned}
& \text { CAT }\left(\mu \mathrm{mol} \mathrm{min} \mathrm{mL}^{-1}\right)=\frac{(\mathrm{A} 240 / \mathrm{min}) \times \text { total volume } \times 1000}{43.6 \times \text { enzyme volume }}
\end{aligned}
$$

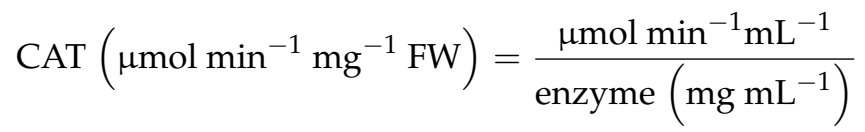

where the absorbance of the sample was recorded by $240 \mathrm{~nm}$ at $1 \mathrm{~min}$, here the extinction coefficient is 43.6 .

\section{Determination of POD}

The POD activity was determined according to the methodology described by Rao et al. [47] and measured by the oxidation of guaiacol at $470 \mathrm{~nm}$ by $\mathrm{H}_{2} \mathrm{O}_{2}$. A reaction mixture of $3 \mathrm{~mL}$ consisted of $0.1 \mathrm{~mL}$ of enzyme extract, $0.05 \mathrm{~mL}$ of $20 \mathrm{mM}$ guaiacol and $2.83 \mathrm{~mL}$ of $10 \mathrm{mM}$ phosphate buffer ( $\mathrm{pH} 7.0$ ). For starting the reaction, $0.02 \mathrm{~mL}$ of $40 \mathrm{mM} \mathrm{H}_{2} \mathrm{O}_{2}$ was added. The mixture with no enzyme extract was considered blank. To reach the temperature equilibration, the blank solution was put in a spectrophotometer for 4 to $5 \mathrm{~min}$. The absorbance reading was taken in the wavelength at $470 \mathrm{~nm}$, and the unit of POD was

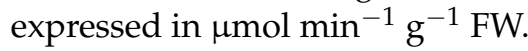

$$
\begin{aligned}
& \text { POD }\left(\mu \mathrm{mol} \mathrm{min}{ }^{-1} \mathrm{~mL}^{-1}\right)=\frac{(\mathrm{A} 470 / \mathrm{min}) \times \text { total volume } \times 1000}{26.6 \times \text { enzyme volume }} \\
& \operatorname{POD}\left(\mu \mathrm{mol} \mathrm{min}{ }^{-1} \mathrm{mg}^{-1} \mathrm{FW}\right)=\frac{\mu \mathrm{mol} \mathrm{min} \mathrm{mL}^{-1}}{\text { enzyme }\left(\mathrm{mg} \mathrm{mL}^{-1}\right)}
\end{aligned}
$$

where the absorbance of the sample was recorded by $470 \mathrm{~nm}$ at $1 \mathrm{~min}$, here the extinction coefficient is 26.6 . 


\subsection{Statistical Analysis}

A randomized complete block design (RCBD) with four replications was adopted. Data were analyzed using SAS 9.4 software. The mean comparison was conducted using Tukey's test at a $5 \%$ probability level.

\section{Conclusions}

The result from this study suggests that allelochemicals present in the methanol extract of $P$. hysterophorus were responsible for the changes of physiological and biochemical parameters of $A$. conyzoides, weedy rice and C. iria. The extract concentrations caused the reduction in chlorophyll content, carotenoids and subsequently hindered the photosynthesis rate. The activity of antioxidant enzymes (SOD, CAT and POD), MDA and proline content was induced and stimulated by foliar spray of $P$. hysterophorus in a concentration-dependent pattern. Ageratum conyzoides maintained comparatively low stress tolerance compared to weedy rice and C. iria. This situation offers the opportunity to control weeds that are resistant to the present herbicides. With the new tools of molecular genetics, proteomics, metabolomics profiling, modern and sophisticated methods of chemistry and biochemistry, we may develop new compounds based on the structure of potential natural herbicidal compounds from P. hysterophorus to formulate selective and eco-friendly herbicides.

Author Contributions: Conceptualization, A.S.J. and M.S.A.H.; methodology, M.S.A.-H. and Z.B.; validation, A.S.J., M.K.U., N.B.A. and M.M.; formal analysis, M.M. and M.H.; investigation, M.M. and M.H.; resources, M.M. and A.S.J.; data curation, M.M. and Z.B.; writing-original draft preparation, M.M.; writing—review and editing, A.K.M.M.I. and Z.B.; visualization, M.M. and M.H.; supervision, A.S.J. and N.B.A.; project administration, A.S.J. and M.M.; funding acquisition, A.S.J. All authors read and confirm the manuscript. All authors have read and agreed to the published version of the manuscript.

Funding: This research received funding from the Research Project entitled "Pest and Disease Monitoring Using Artificial Intelligent for Risk Management of Rice Under Climate Change" under Long-term Research Grant Scheme (LRGS), Ministry of Higher Education, Malaysia (LRGS/1/2019/UPM/ /2; vote number: 5545002), Fundamental Research Grant Scheme (FRGS), Malaysia (FRGS/1/2017/WAB01/ UPM/01/2) and Putra Grant UPM (GP-IPB/2017/9523400).

Institutional Review Board Statement: Not applicable.

Informed Consent Statement: Not applicable.

Data Availability Statement: The data presented in this study are available in the article.

Acknowledgments: The authors are grateful to the Research Project entitled "Pest and Disease Monitoring Using Artificial Intelligent for Risk Management of Rice Under Climate Change" under Long-term Research Grant Scheme (LRGS), Ministry of Higher Education, Malaysia (LRGS/1/2019/UPM/ /2; vote number: 5545002), Fundamental Research Grant Scheme (FRGS), Malaysia (FRGS/1/2017/WAB01/ UPM/01/2) and Putra Grant UPM (GP-IPB/2017/9523400) also sincerely acknowledges the University Putra Malaysia for providing facilities. The authors would like to thank Nik Amelia Nik Mustapha for her assistance during this experiment conducted. We also express gratitude to Md. Parvez Anwar, for editing the English of this manuscript.

Conflicts of Interest: The authors declare no conflict of interest.

\section{References}

1. Batish, D.R.; Kaur, S.; Singh, H.P.; Kohli, R.K. Nature of interference potential of leaf debris of Ageratum conyzoides. Plant Growth Regul. 2008, 57, 137-144. [CrossRef]

2. Song, B.-K.; Chuah, T.-S.; Tam, S.M.; Olsen, K.M. Malaysian weedy rice shows its true stripes: WildOryzaand elite rice cultivars shape agricultural weed evolution in Southeast Asia. Mol. Ecol. 2014, 23, 5003-5017. [CrossRef] [PubMed]

3. Van Chin, D. Biology and management of barnyardgrass, red sprangletop and weedy rice. Weed Biol. Manag. 2001, 1, 37-41. [CrossRef]

4. Chauhan, B.S. Strategies to manage weedy rice in Asia. Crop. Prot. 2013, 48, 51-56. [CrossRef]

5. Nadir, S.; Xiong, H.-B.; Zhu, Q.; Zhang, X.-L.; Xu, H.-Y.; Li, J.; Dongchen, W.; Henry, D.; Guo, X.-Q.; Khan, S.; et al. Weedy rice in sustainable rice production. A review. Agron. Sustain. Dev. 2017, 37, 46. [CrossRef] 
6. Ismail, B.S.; Siddique, M.A.B. The Inhibitory Effect of Grasshopper's Cyperus (Cyperus iria L.) on the Seedling Growth of Five Malaysian Rice Varieties. Trop. Life Sci. Res. 2011, 22, 81-89.

7. Srinivas, C.; Lakshmi, C. Parthenium: A wide angle view. Indian J. Dermatol. Venereol. Leprol. 2007, 73, 296-306. [CrossRef]

8. Belz, R.G. Allelopathy in crop/weed interactions-An update. Pest Manag. Sci. 2007, 63, 308-326. [CrossRef] [PubMed]

9. Adkins, S.W.; Sowerby, M.S. Allelopathic potential of the weed, Parthenium hysterophorus L. in Australia. Plant Prot. Q. 1996, $11,20-23$.

10. Motmainna, M.; Juraimi, A.S.; Uddin, M.K.; Asib, N.B.; Islam, A.K.M.M.; Hasan, M. Allelopathic potential of Malaysian invasive weed species on Weedy rice (Oryza sativa f. spontanea Roshev). Allelopathy J. 2021, 53, 53-68. [CrossRef]

11. Kaya, A.; Yigit, E. The physiological and biochemical effects of salicylic acid on sunflowers (Helianthus annuus) exposed to flurochloridone. Ecotoxicol. Environ. Saf. 2014, 106, 232-238. [CrossRef]

12. Gill, S.S.; Tuteja, N. Reactive oxygen species and antioxidant machinery in abiotic stress tolerance in crop plants. Plant Physiol. Biochem. 2010, 48, 909-930. [CrossRef]

13. Maoka, T. Carotenoids as natural functional pigments. J. Nat. Med. 2020, 74, 1-16. [CrossRef]

14. Shah, S.H.; Houborg, R.; McCabe, M.F. Response of Chlorophyll, Carotenoid and SPAD-502 Measurement to Salinity and Nutrient Stress in Wheat (Triticum aestivum L.). Agronomy 2017, 7, 61. [CrossRef]

15. Hasanuzzaman, M.; Bhuyan, M.B.; Zulfiqar, F.; Raza, A.; Mohsin, S.M.; Al Mahmud, J.; Fujita, M.; Fotopoulos, V. Reactive Oxygen Species and Antioxidant Defense in Plants under Abiotic Stress: Revisiting the Crucial Role of a Universal Defense Regulator. Antioxidants 2020, 9, 681. [CrossRef]

16. Sheng, Y.; Abreu, I.A.; Cabelli, D.E.; Maroney, M.J.; Miller, A.-F.; Teixeira, M.; Valentine, J.S. Superoxide Dismutases and Superoxide Reductases. Chem. Rev. 2014, 114, 3854-3918. [CrossRef]

17. Li, D.-H.; Yuan, Y. Hydrogen peroxide enhances antioxidative defense in the leaves of water caltrop (Trapa bicornis) seedlings treated with lead. Biologia 2016, 71, 100-108. [CrossRef]

18. Ayala, A.; Munoz, M.F.; Argüelles, S. Lipid peroxidation: Production, metabolism, and signaling mechanisms of malondialdehyde and 4-hydroxy-2-nonenal. Oxid. Med. Cell. Long. 2014, 1-31. [CrossRef]

19. Catalá, A.; Díaz, M. Editorial: Impact of Lipid Peroxidation on the Physiology and Pathophysiology of Cell Membranes. Front. Physiol. 2016, 7, 423. [CrossRef]

20. Khayatnezhad, M.; Gholamin, R.; Jamaati-e-Somarin, S.; Zabihi-e-Mahmoodabad, R. The leaf chlorophyll content and stress resistance relationship considering in corn cultivars (Zea mays). Adv. Environ. Biol. 2011, 5, 118-122.

21. Ding, H.; Cheng, Z.; Liu, M.; Hayat, S.; Feng, H. Garlic exerts allelopathic effects on pepper physiology in a hydroponic co-culture system. Biol. Open 2016, 5, 631-637. [CrossRef]

22. Y1lmaz, E.; Tuna, A.L.; Bürün, B. Tolerance strategies developed by plants to the effects of salt stress. CBU J. Sci. 2011, 7, 47-66.

23. Erdal, S. Androsterone-induced molecular and physiological changes in maize seedlings in response to chilling stress. Plant Physiol. Biochem. 2012, 57, 1-7. [CrossRef]

24. Mishra, S.; Srivastava, S.; Tripathi, R.; Govindarajan, R.; Kuriakose, S.; Prasad, M. Phytochelatin synthesis and response of antioxidants during cadmium stress in Bacopa monnieri L. Plant Physiol. Biochem. 2006, 44, 25-37. [CrossRef]

25. El-Shora, H.M.; Abd El-Gawad, A.M. Physiological and biochemical responses of Cucurbita pepo L. mediated by Portulaca oleracea L. allelopathy. Fresenius Environ. Bull. 2015, 24, 386-393.

26. Lawlor, D.W. Limitation to Photosynthesis in Water-stressed Leaves: Stomata vs. Metabolism and the Role of ATP. Ann. Bot. 2002, 89, 871-885. [CrossRef]

27. Foyer, C.H.; Shigeoka, S. Understanding Oxidative Stress and Antioxidant Functions to Enhance Photosynthesis. Plant Physiol. 2011, 155, 93-100. [CrossRef] [PubMed]

28. Casson, S.; Gray, J.E. Influence of environmental factors on stomatal development. New Phytol. 2008, 178, 9-23. [CrossRef] [PubMed]

29. Blum, U.; Gerig, T.M. Relationships between Phenolic Acid Concentrations, Transpiration, Water Utilization, Leaf Area Expansion, and Uptake of Phenolic Acids: Nutrient Culture Studies. J. Chem. Ecol. 2005, 31, 1907-1932. [CrossRef]

30. Li, Z.-H.; Wang, Q.; Ruan, X.; Pan, C.-D.; Jiang, D.-A. Phenolics and Plant Allelopathy. Molecules 2010, 15, 8933-8952. [CrossRef]

31. Rakhra, G.; Sharma, A.D.; Singh, J. Anti-oxidative potential of boiling soluble antioxidant enzymes inAmelioration of droughtinduced oxidative stress in tolerant and sensitive cultivars of Triticum aestivum. J. Crop. Sci. Biotechnol. 2015, 18, 103-122. [CrossRef]

32. Quan, L.-J.; Zhang, B.; Shi, W.-W.; Li, H.-Y. Hydrogen Peroxide in Plants: A Versatile Molecule of the Reactive Oxygen Species Network. J. Integr. Plant Biol. 2008, 50, 2-18. [CrossRef] [PubMed]

33. Saidi, I.; Ayouni, M.; Dhieb, A.; Chtourou, Y.; Chaïbi, W.; Djebali, W. Oxidative damages induced by short-term exposure to cadmium in bean plants: Protective role of salicylic acid. S. Afr. J. Bot. 2013, 85, 32-38. [CrossRef]

34. Choudhury, S.; Panda, S.K. Role of salicylic acid in regulating cadmium induced oxidative stress in Oryza sativa L. roots. Bulg. J. Plant Physiol. 2004, 30, 95-110. [CrossRef]

35. Zandalinas, S.I.; Mittler, R.; Balfagón, D.; Arbona, V.; Gómez-Cadenas, A. Plant adaptations to the combination of drought and high temperatures. Physiol. Plant. 2018, 162, 2-12. [CrossRef] [PubMed]

36. Wani, A.; Hayat, S.; Ahmad, A.; Tahir, I. Efficacy of brassinosteroid analogues in the mitigation of toxic effects of salt stress in Brassica juncea plants. J. Environ. Biol. 2017, 38, 27-36. [CrossRef] 
37. Abbas, W.; Ashraf, M.; Akram, N.A. Alleviation of salt-induced adverse effects in eggplant (Solanum melongena L.) by glycinebetaine and sugarbeet extracts. Sci. Hortic. 2010, 125, 188-195. [CrossRef]

38. El-Khatib, A.A.; Barakat, N.A.; Nazeir, H. Growth And Physiological Response Of Some Cultivated Species under Allelopathic Stress of Calotropis Procera (Aiton) WT. Appl. Sci. Rep. 2016, 14, 237-246. [CrossRef]

39. Motmainna, M.; Juraimi, A.S.; Uddin, M.K.; Asib, N.B.; Islam, A.K.M.M.; Hasan, M. Bioherbicidal Properties of Parthenium hysterophorus, Cleome rutidosperma and Borreria alata Extracts on Selected Crop and Weed Species. Agronomy 2021, 11, 643. [CrossRef]

40. El-Mergawi, R.A.; Al-Humaid, A.I. Searching for natural herbicides in methanol extracts of eight plant species. Bull. Natl. Res. Cent. 2019, 43, 1-6. [CrossRef]

41. Lichtenthaler, H.K.; Buschmann, C. Chlorophylls and Carotenoids: Measurement and Characterization by UV-VIS Spectroscopy. Curr. Protoc. Food Anal. Chem. 2001, 1, F4.3.1-F4.3.8. [CrossRef]

42. Amin, T.M. Response to Salt Stress and Strategies to Improve Salt Tolerance in Chinese Kale (Brassica Oleracea var. Albo-Glabra). Ph.D. Thesis, University Putra Malaysia, Seri Kembangan, Malaysia, 2012.

43. Stewart, R.R.C.; Bewley, J.D. Lipid Peroxidation Associated with Accelerated Aging of Soybean Axes. Plant Physiol. 1980, 65, 245-248. [CrossRef] [PubMed]

44. Bates, L.S.; Waldren, R.P.; Teare, I.D. Rapid determination of free proline for water-stress studies. Plant Soil 1973, 39, 205-207. [CrossRef]

45. Gupta, A.S.; Heinen, J.L.; Holaday, A.S.; Burke, J.J.; Allen, R.D. Increased resistance to oxidative stress in transgenic plants that overexpress chloroplastic Cu/Zn superoxide dismutase. Proc. Natl. Acad. Sci. USA 1993, 90, 1629-1633. [CrossRef]

46. Aebi, H. Catalase in vitro. Methods Enzymol. 1984, 105, 121-126. [CrossRef] [PubMed]

47. Rao, M.V.; Paliyath, G.; Ormrod, D.P. Ultraviolet-B-and ozone-induced biochemical changes in antioxidant enzymes of Arabidopsis thaliana. Plant Physiol. 1996, 110, 125-136. [CrossRef] 